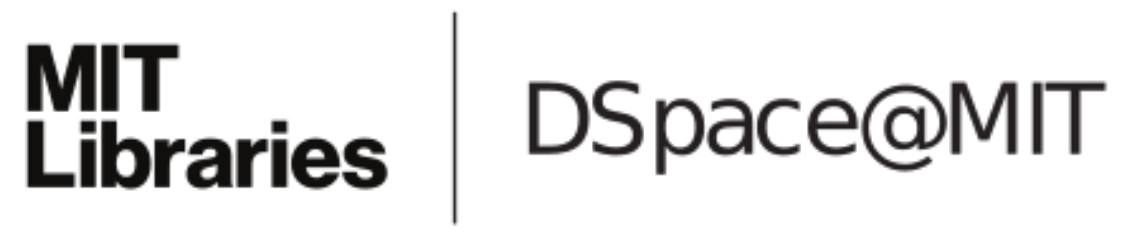

\author{
MIT Open Access Articles
}

Probabilities of conditionals in context

The MIT Faculty has made this article openly available. Please share how this access benefits you. Your story matters.

Citation: Khoo, Justin. "Probabilities of Conditionals in Context." Linguistics and Philosophy 39.1 (2016): 1-43.

As Published: http://dx.doi.org/10.1007/s10988-015-9182-z

Publisher: Springer Netherlands

Persistent URL: http://hdl.handle.net/1721.1/103518

Version: Author's final manuscript: final author's manuscript post peer review, without publisher's formatting or copy editing

Terms of use: Creative Commons Attribution-Noncommercial-Share Alike 


\title{
Probabilities of conditionals in context
}

\author{
Justin Khoo \\ jkhoo@mit.edu \\ Forthcoming, Linguistics \& Philosophy
}

\begin{abstract}
The Ramseyan thesis that the probability of an indicative conditional is equal to the corresponding conditional probability of its consequent given its antecedent is both widely confirmed and subject to attested counterexamples (e.g., [mcgee:2000, kaufmann:2004]). This raises several puzzling questions. For instance, why are there interpretations of conditionals that violate this Ramseyan thesis in certain contexts, and why are they otherwise very rare? In this paper, I raise some challenges to Stefan Kaufmann's account of why the Ramseyan thesis sometimes fails, and motivate my own theory. On my theory, the proposition expressed by an indicative conditional is partially determined by a background partition, and hence its probability depends on the choice of such a partition. I hold that this background partition is contextually determined, and in certain conditions is set by a salient question under discussion in the context. I show how the resulting theory offers compelling answers to the puzzling questions raised by failures of the Ramseyan thesis.
\end{abstract}

Few quotes in the literature on indicative conditionals are as famous, and have been as influential, as the following remark of Frank Ramsey's:

If two people are arguing 'If p will q?' and both are in doubt as to $\mathrm{p}$, they are adding $\mathrm{p}$ hypothetically to their stock of knowledge and arguing on that basis about $\mathrm{q}$... We can say that they are fixing their degrees of belief in q given p. ([ramsey:1931], p. 249)

Ramsey's observation has been subject to a variety of interpretations, but the core idea seems to be something like the following (restricting ourselves to indicative conditionals for the scope of this paper): ${ }^{1}$

\footnotetext{
${ }^{1}$ Of course, competent speakers often err in their probability judgments, so really this ought to be a normative claim about what the probability of a conditional ought to be (given the relevant evidence). I won't worry about distinguishing the normative/descriptive versions of RAMSEY's OBSERVATION in what follows.
} 
RAMSEY'S OBSERVATION: For any conditional, the probability that it is true is equal to the conditional probability that its consequent is true, given that its antecedent is.

(Where the conditional probability that $C$, given $A$ is the probability that $C$ ought to have were one to learn $A$ with certainty and nothing else.)

RAMSEY'S OBSERVATION is both widely endorsed - for instance, [stalnaker:1970, adams:1975, fraassen:1976, gibbard:1981, jeffrey:1991, edgington:1995, bennett:2003] each construct semantic theories of indicative conditionals which aim to predict RAMSEY'S OBSERVATION ${ }^{2}$ and extremely well-confirmed. For example, consider the ease with which we move back and forth between talk of probabilities of conditionals and conditional likelihood:

a. The likelihood that John came to the party if Sue did is high.

b. The likelihood that John came to the party given Sue did is high.

Or, suppose that John has just rolled a fair 12-sided die and kept the result hidden. Now consider the following conditional, and assume for now that 1 is not a prime number:

If John rolled a prime number, then he rolled an odd number.

It seems correct that the probability that (2) is true is high, and the reason its probability is high is because the corresponding conditional probability that John rolled an odd, given that he rolled a prime, is high (5/6) - just as RAMSEY'S OBSERVATION predicts. ${ }^{3}$

Nonetheless, there are attested counterexamples to RAMSEY'S OBSERVATION in the literature (for instance, see [pollock:1981, mcgee:2000] and most recently [kaufmann:2004]). This is puzzling: we have a well-confirmed hypothesis about conditionals and conditional probability that nonetheless seems false due to some complex counterexamples. What should we think about this puzzle? Theories that take RAMSEY's OBSERVATion as their starting point and use it to characterize the meanings of indicative conditionals (for instance, the "probabilities-first" theories of [adams:1975, edgington:1995, bennett:2003])

\footnotetext{
${ }^{2}$ Even most of those who reject the letter of RAMSEY'S OBSERVATION accept the spirit of it-for instance, material conditional theorists like [lewis:1976, jackson:1979] reject RAMSEY'S OBSERVATION but hold that the assertability (though not probability of truth) of a conditional is governed by its corresponding conditional probability.

${ }^{3}$ Empirical studies confirm RAMSEY'S OBSERVATION as well. [evans:2004, douven:2013] found a high correlation between subjects' judgments of the probability of a conditional and its corresponding conditional probability.
} 
must argue that its alleged counterexamples are illegitimate, for such theories lack the resources to predict exceptions to it. ${ }^{4}$ However, as we'll see in $\S 1$, there are good reasons to think the counterexamples are genuine. Thus, our task is to find a theory that (a) predicts such counterexamples, and (b) explains why such counterexamples are rare. My goal in this paper is to motivate just such a theory of conditionals.

The rest of this paper is structured as follows. In $\S 1$, I motivate two different scenarios which give rise to Ramsey-violating conditionals, highlighting some puzzling observations about the data to be explained. In $\S 2$, I turn to [kaufmann:2004]'s theory, which is as far as I know the only theory flexible enough to predict both Ramsey-friendly and Ramseyviolating interpretations of conditionals. Unfortunately, as I will argue in that section, Kaufmann's theory faces several challenges, motivating us to look elsewhere for a suitable theory. I motivate my own theory in the rest of the paper. In $\S 3$, I argue that the difference between Ramsey-friendly and Ramsey-violating conditionals must lie in how their probabilities are distributed at regions of epistemic space where their antecedents are false. This prompts me to explore a semantics for conditionals in $\S 4$ in which conditionals have non-trivial probabilities when their antecedents are false. In that section, I show how a generalization of a Stalnakerian closest-worlds semantics of conditionals can incorporate partition dependence, and hence promises a unified theory of Ramsey-friendly and Ramseyviolating interpretations of conditionals. In $\S 5$, I motivate a pragmatic theory governing the partitions conditionals are interpreted relative to. I show that the resulting theory offers a compelling explanation of our intuitions about Ramsey-friendly and Ramsey-violating conditionals in a range of cases. Finally, I close in $\S 6$ with a discussion of how to integrate my truth-conditional semantics with a theory of indeterminacy to yield the right probabilities of conditionals.

\footnotetext{
${ }^{4}$ Interestingly, [kratzer:1986, kratzer:2012]'s theory, on which if-clauses restrict the domains of modals and quantifiers, may also be unable to predict such exceptions to RAMSEY'S OBSERVATION. On one understanding of Kratzer's proposal, judging the probability that some conditional sentence is true just is to judge the probability that its consequent is true, using a probability measure whose domain is restricted (conditionalized) on the conditional's antecedent. This understanding of her proposal will predict no exceptions to RAMSEY'S OBSERVATION. However, evidence that if-clauses do not always restrict overt modals/quantifiers (cf. [frank:1997, geurts:2004, geurts:2005]) may motivate a version of Kratzer's view on which there are two ways to judge the probability that some conditional sentence is true: the way described above, and another way, on which one judges the probability of the proposition expressed by the conditional sentence. On this version of Kratzer's view, exceptions to RAMSEY'S OBSERVATION may be predicted. However, since this latter view will only generate plausible exceptions to RAMSEY'S OBSERVATION given something like the kind of partition dependent semantics discussed in this paper, I won't discuss it further at this time.
} 


\section{Counterexamples to RAMSEY'S OBSERVATION}

Here's a version of Kaufmann's “two urns" case:

Hospital. You are an administrator in a regional hospital tasked with sorting incoming patients. A recent outbreak of B-flu in the region has led to a flood of new patients. Currently, there are 72 patients in the waiting room: 60 from the town of Appleton, and 12 from the town of Zephyr. Here is a summary of what you know about the patients in the waiting room:

\begin{tabular}{ll} 
Appleton patients & Zephyr patients \\
\hline 60 total & 12 total \\
10 showing symptoms & 10 showing symptoms \\
$\circ 1$ with B-flu & $\circ 9$ with B-flu \\
$\circ 9$ without B-flu & $\circ 1$ without B-flu \\
50 not showing symptoms & 2 not showing symptoms \\
$\circ$ all without B-flu & $\circ$ all without B-flu
\end{tabular}

Given what you know, learning of a patient that he or she shows symptoms of B-flu means that patient has about a $63 \%$ chance of being from Zephyr rather than Appleton.

You are checking patients in, directing them their respective doctors. Currently, 24 patients have been checked in, all from Appleton. Therefore, there is a $75 \%$ chance that the next patient is from Appleton. You see that his name is Jones.

Let $S$ be the proposition that Jones is showing symptoms of B-flu, $B$ be the proposition that Jones has B-flu, $Z$ the proposition that Jones is from Zephyr, and $\bar{Z}$ the proposition that Jones is not from Zephyr (which is equivalent by assumption with the proposition that Jones is from Appleton). Summarizing the relevant probabilities: ${ }^{5}$

$$
P(Z)=1 / 4
$$

\footnotetext{
${ }^{5} \mathrm{I}$ assume that a probability function $P$ is any function from subsets of $\mathcal{W}$ (the propositions) to values in $[1,0]$ that obeys the Kolmogorov axioms:

1. $P(A) \geq 0$, for all $p \in \wp(\mathcal{W})$

2. $P(\mathcal{W})=1$

3. $P(A \cup B)=P(A)+P(B)$, for all $p, q \in \wp(\mathcal{W})$ such that $A \cap B=\emptyset$

plus the following definition for conditional probabilities (I follow the standard convention of letting ' $A B$ ' denote the intersection of $A$ and $B$ and let ' $\bar{A}$ ' denote $W \backslash A$ ):

4. $P(B \mid A)=\frac{P(A B)}{P(A)}$, where $P(A)>0$
} 


$$
\begin{aligned}
& P(S)=1 / 3 \\
& P(Z \mid S)=5 / 8 \\
& P(B \mid S Z)=9 / 10
\end{aligned}
$$

Question: given this information, how likely do you find the following claim?

Given that Jones is most likely from Appleton, and that people from Appleton showing symptoms of B-flu most likely do not have B-flu, it seems that (3) is unlikely to be true. However, now suppose that you learn that Jones has symptoms of B-flu. How likely ought you to think that Jones has B-flu? Having just learned this new information, it seems that you ought to think Jones is more likely from Zephyr than Appleton, since most people showing symptoms of B-flu are from Zephyr. But if he's from Zephyr and showing symptoms, it's likely that Jones has B-flu, since 9 out of 10 people from Zephyr who show signs of B-flu have it. Therefore, in a case like Hospital, the probability of (3) is low even though the corresponding conditional probability of its consequent given its antecedent is high, or at least fifty-fifty. Thus given the information in Hospital, (3) is a prima facie counterexample to RAMSEY's OBSERVATION. ${ }^{6}$

\footnotetext{
${ }^{6}$ The cases in [mcgee:2000, kaufmann:2004] are structurally analogous to Hospital. Another class of counterexamples are due to [fraassen:1980] (attributed to Richmond Thomason; see also [lewis:1986a, jackson:1987, bennett:2003]):

(i) a. If Sally is deceiving me, I don't believe it.

b. If my business partner is cheating me, I will never find out.

However, there is a difference between these counterexamples and the McGee/Kaufmann counterexamples. The Thomason examples seem acceptable only on their 'concessive' reading, on which the speaker is already certain of the consequent. Thus, contrast the oddity of the following:

(ii) a. \#I'm not sure whether I believe Sally is deceiving me, but if she is, I don't believe it. b. $\quad$ \#I might find out my partner is cheating me, but if he is, I will never find out.

with the acceptability of (which does not require a concessive reading):

(iii) Jones might not have B-flu, but if he's showing symptoms of B-flu, he has it.

This preliminary observation suggests that something different is going on with the Thomason counterexamples than with the McGee/Kaufmann examples discussed below. However, a full discussion of the Thomason examples is beyond the scope of this paper, and I will set them aside in what follows. See [chalmers:2007, willer:2010] for further discussion.
} 
Now, admittedly, there is a lot of probabilistic information in the description of HosPITAL, which may tempt us to think that our intuitions in this case are untrustworthy. However, it seems unlikely that the Ramsey-violating intuition about (3) in Hospital is due to some sort of cognitive mistake. ${ }^{7}$ Furthermore, less complex (but structurally similar, as we'll see below) counterexamples to RAMSEY'S OBSERVATION exist as well. To begin, notice that $P(B \mid A)$ does not care about what happens to $B$ at the $\bar{A}$-region of logical space (since neither $P(B A)$ and $P(A)$ do). Thus, if $B$ and $C$ are epistemically equivalent at the $A$-region of epistemic space, $P(B \mid A)=P(C \mid A)$. Therefore, if RAMSEY's OBSERvation is without exception, then whenever $P(B \equiv C \mid A)=1, A \rightarrow B$ and $A \rightarrow C$ must be equiprobable. But there are intuitive counterexamples to this consequence: ${ }^{8}$

Oracle. A highly reliable oracle is about to tell us whether a recently-tossed coin landed heads or tails. Since you know the coin to be weighted towards tails, you are pretty sure it landed tails. The oracle then makes her prediction, although you can't quite make out what she says. You are pretty sure she says "heads".

a. If the oracle is correct, the coin landed heads.

b. If the oracle is correct, then she said "heads".

It seems plausible in this case that (4-a) and (4-b) are not equiprobable. Remember, you know that the oracle is reliable and think she most likely said "heads". So, most naturally, (4-a) is likely true. But of course you also know that the oracle is reliable and think the coin most likely landed tails (because it is biased). So, most naturally, (4-b) is unlikely true (since you think that if the oracle is correct, she most likely said "tails"). However, notice that the proposition that the coin landed heads and the proposition that the oracle said "heads" are equivalent under the assumption that the oracle is correct. Therefore, were RAMSEY'S OBSERVATION without exception, (4-a) and (4-b) should be equiprobable, contrary to our intuitions in the case! ${ }^{9}$

\footnotetext{
${ }^{7}$ For instance, [rothschild:2013a] suggests the possibility that the mistake is akin to base rate neglect-a tendency to ignore general information in favor of specific information (cf. [kahneman:1973, kahneman:1982, barhillel:1980]). However, if this were what happens when we judge the probability of (3) in Hospital, then we should expect the mistake to be correctable, perhaps by presenting the information using natural frequencies with respect to an intuitive reference class (cf. [gigerenzer:1995, hoffrage:2000]). But notice that the relevant information in Hospital, the proportion of patients showing symptoms of B-flu from Appleton/Zephyr, is already presented using natural frequencies as well as probabilities. So we should already be correcting for the mistake given how the information is presented in the case!

${ }^{8}$ I owe this example to Steve Yablo (personal communication).

${ }^{9}$ Here's another way to put the lesson from Oracle. Observe that we do not have clear intuitions in Oracle about the conditional probability of the coin landing heads given that the oracle is correct. After
} 
Complicating the data is [kaufmann:2004]'s observation that even in a context like Hospital, it is possible to bias a Ramsey-friendly interpretation of (3) by making it the conclusion of a probability-preserving argument as follows (p588):

$$
\text { If Jones is showing symptoms of B-flu, then he is from Zephyr. }
$$

But then if he is showing symptoms of B-flu and is from Zephyr, he has B-flu.

Therefore,

(3) If Jones is showing symptoms of B-flu, he has B-flu.

Notice that the reasoning from (5) and (6) to (3) instantiates a probabilistically valid rule of inference; so, if (5) and (6) are together very likely, then (3) must also be likely. But intuitively it does seem that (5) and (6) are together quite likely. Indeed, in the context of this argument, the pull to say that (3) is likely is undeniable - just as it is hard to resist the pull to say that (3) is unlikely when initially presented with the case. Thus, it seems that the Ramsey-violating intuition in cases like Hospital is fickle - it can be overridden by altering certain features of the context. ${ }^{10}$

This data is puzzling. In particular, we'd like to know:

a. What is the nature of the Ramsey-friendly and Ramsey-violating interpretations of

all, suppose you learn that the oracle is correct - then you know that something you previously thought was likely is false. But, given the description of the case, it is unclear what you should think is more likely in such a circumstance - that the oracle in fact said "tails" or that the coin in fact landed heads. This is reminiscent of Gibbard's "Sly Pete" case (cf. [gibbard:1981, stalnaker:1984, edgington:1997, bennett:2003, williams:2008]), though "Sly Pete" cases are often taken to motivate a non-propositional "probabilitiesfirst" theory of indicative conditionals. Here, we instead have a problem for RAMSEY'S OBSERVATION, since we don't have clear intuitions about the conditional probability corresponding to either conditional (notice the corresponding conditional probabilities are equivalent), yet we have a clear intuition that (4-a) is likely and (4-b) is not. Furthermore, it is not obvious how to apply the strategy of explaining away our intuitions in Oracle as a cognitive illusion. For instance, the base rate neglect strategy discussed in footnote 7 claims that we're ignoring some general information in favor of specific information when we form our probability judgments in the case. But what general information are we supposed to be ignoring when we evaluate the probabilities of (4-a) and (4-b) in Oracle?

${ }^{10} \mathrm{An}$ anonymous reviewer points out that something similar can be done for Oracle:

(i) a. If the oracle is correct, the coin landed heads.

b. If the oracle is correct, then the coin landed heads if and only if she said 'heads'.

c. So, if the oracle is correct, she said 'heads'.

In the context of this argument, it's clear that (4-a) and (4-b) should be equally likely, but not clear what probability either has. 
conditionals? (Is it an ambiguity, for instance?)

b. Why do certain conditionals favor Ramsey-violating interpretations in certain contexts?

c. Why, in such contexts, can we sometimes override the Ramsey-violating interpretations in favor of Ramsey-friendly interpretations?

d. Why are Ramsey-violating interpretations of conditionals so rare?

These are the questions with which I will be concerned in this paper. In the next section, I will sketch [kaufmann:2004]'s theory, which predicts both Ramsey-friendly and Ramseyviolating interpretations of conditionals (and is, as far as I know, the only theory on the market to do so). After proposing some challenges to Kaufmann's theory, I will argue for my own view in the rest of the paper.

\section{Kaufmann's probabilistic theory}

The basic idea behind [kaufmann:2004]'s theory is that there are two ways of interpreting a conditional ("locally" and "globally") relative to a background partition set in the context. Rather than provide a truth conditional semantics for conditionals that generates these two interpretations, Kaufmann shows us how to distinguish them by seeing how their probabilities are calculated. The probability of the local interpretation of a conditional $A \rightarrow_{\ell} B$ is calculated by distributing the corresponding conditional probability of $B$ given $A$ across the cells of the background partition. Since the background partition for (3) is $\mathbf{Z}$ $(=\{Z, \bar{Z}\})$ in Hospital, we have:

$$
\begin{aligned}
& P\left(S \rightarrow_{\ell}^{\mathbf{Z}} B\right)=P(B \mid S Z) \cdot P(Z)+P(B \mid S \bar{Z}) \cdot P(\bar{Z}) \\
& =9 / 10 \cdot 1 / 4+1 / 10 \cdot 3 / 4 \\
& =9 / 40+3 / 40 \\
& =3 / 10
\end{aligned}
$$

Local

The probability of the global interpretation of a conditional $A \rightarrow_{g} B$ is calculated by distributing the corresponding conditional probability of $B$ given $A$ across the cells of the relevant background partition after conditionalizing each cell on $A$. This, it turns out, is equal to the corresponding conditional probability of the conditional's consequent on its antecedent: 


$$
\begin{aligned}
& P\left(S \rightarrow{ }_{g}^{\mathbf{Z}} B\right)=(B \mid S)=P(B \mid S Z) \cdot P(Z \mid S)+P(B \mid S \bar{Z}) \cdot P(\bar{Z} \mid S) \\
& =\quad 9 / 10 \cdot 5 / 8+1 / 10 \cdot 3 / 8 \\
& =\quad 45 / 80+3 / 80 \\
& =\quad 3 / 5
\end{aligned}
$$

Thus, Kaufmann's theory predicts that the same conditional sentence can have both Ramsey-friendly and Ramsey-violating interpretations. This is a major advance for understanding the counterexamples to RAMSEY'S OBSERVATION from the previous section. But what is the nature of the background partition conditionals are interpreted relative to? Drawing on [skyrms:1988, skyrms:1990], Kaufmann proposes that it is an objectivechance determining variable $\mathbf{Z}$ which partitions the space of epistemic possibilities and is such that (see [kaufmann:2004]: 595-597):

1. The objective chance of $B$ depends on $A$ and $\mathbf{Z}$,

2. $\mathbf{Z}$ is causally independent of $A$, and

3. $\mathbf{Z}$ is evidentially dependent on $A$.

As mentioned above, Kaufmann's theory marks a significant advance in our thinking about conditionals. However, I think it faces some challenges. First, it is incomplete: since Kaufmann never specifies a semantics for conditionals, it remains unclear why conditionals should have "local" and "global" interpretations - for instance, is it due to ambiguity or perhaps context-dependence? ${ }^{11}$ Second, while Kaufmann's theory predicts these two interpretations, it does not explain why the Ramsey-violating "local" interpretation of (3) is favored by default in Hospital, even though in the context of the argument with premises (5) and (6), it seems to favor its Ramsey-friendly "global" interpretation. Third and relatedly,

\footnotetext{
${ }^{11}$ Kaufmann himself seems to waver between these two ways of understanding his proposal. For instance, [kaufmann:2004] suggests that the two probabilities are due to an ambiguity in conditionals (p. 603), and [rothschild:2013a] also seems to endorse this position (p. 16). However, see [kaufmann:2009a] for a suggestion that the difference is "pragmatic" (p. 26-27) although I find that suggestion hard to make sense of. Here is the relevant quote from p.26:

"The pragmatic account, in contrast, assumes that the very same conditional is interpreted with respect to different probability distributions ... I call it 'pragmatic' because it appeals to the inference involved in evaluating the conditional, corresponding to different ways of weighing the values assigned according to (21)."
} 
in the Oracle case, both conditionals (4-a) and (4-b) seem to favor Ramsey-violating interpretations. To predict the right result, on Kaufmann's theory they must be interpreted relative to different partitions ((4-a) relative to whether the oracle said "heads" $\mathbf{S}_{\mathbf{H}}$, and (4-b) relative to whether the coin landed heads $\mathbf{H}$ ). However, nothing in Kaufmann's pragmatics explains why this should be the case. ${ }^{12}$ Thus, Kaufmann's theory is silent about our questions (a)-(c).

Fourth, condition 2 of Kaufmann's theory of background partitions also seems to be false. There are cases of Ramsey-violating conditionals whose background partition is intuitively one which is causally dependent on $A$. We can see this by keeping the numbers from Hospital but modifying the presentation of the information as follows:

Smoking. You are an administrator in a regional hospital tasked with sorting incoming patients. You know that smoking in one's twenties is a reliable (but not deterministic) cause of cancer later in life. You also know that smoking in one's twenties is positively correlated with having gene $\mathrm{X}$ in people with cancer, and negatively correlated with having gene $\mathrm{X}$ in people without cancer. Currently, there are 72 elderly patients in the waiting room: 60 without cancer, and 12 with cancer. Here is a summary of what you know about the patients in the waiting room:

You are checking patients in, directing them their respective doctors. Currently, 24 patients have been checked in, all without cancer. So, there is a $1 / 3$ chance the next patient has cancer. You see that his name is Jones.

\footnotetext{
${ }^{12}$ Both partitions are causally independent but evidentially dependent on the oracle being correct. Focus on (4-a) ('if the oracle is correct, the coin landed heads') to keep things simple - analogous reasoning will apply to (4-b). Either the objective chance of the coin landing heads depends on the oracle being correct and whether the oracle said "heads" or it does not. If it does not, then Kaufmann's theory predicts that (4-a) will not be interpreted relative to the partition whether the oracle said "heads" (since it violates condition 1). If it does, then it should also be the case that the objective chance of the coin landing heads depends on the oracle being correct and whether the coin lands heads - in fact, it is even more obvious that this is so! But then, according to Kaufmann's theory, two distinct partitions meet all the criteria to be the partition that (4-a) is interpreted relative to in Oracle. And whether (4-a) is interpreted relative to one rather than the other makes a difference to its "local" interpretation:
}

$$
\begin{aligned}
P\left(O \rightarrow{ }_{\ell}^{\mathbf{S}_{\mathbf{H}}} H\right)= & \\
& P\left(H \mid S_{H} O\right) \cdot P\left(S_{H}\right)+P\left(H \mid \overline{S_{H}} O\right) \cdot P\left(\overline{S_{H}}\right)= \\
& 1 \cdot P\left(S_{H}\right)+0 \cdot P\left(\overline{S_{H}}\right)= \\
& P\left(S_{H}\right)
\end{aligned}
$$

$$
\begin{aligned}
P\left(O \rightarrow{ }_{\ell}^{\mathbf{H}} H\right)= & \\
& P(H \mid H O) \cdot P(H)+P(H \mid \bar{H} O) \cdot P(\bar{H})= \\
& 1 \cdot P(H)+0 \cdot P(\bar{H})= \\
& P(H)
\end{aligned}
$$

Only if interpreted relative to $\mathbf{S}_{\mathbf{H}}$ is (4-a) likely true. 


\begin{tabular}{ll} 
No cancer & Cancer \\
\hline 60 total & 12 total \\
10 who smoked in their twenties & 10 who smoked in their twenties \\
$\circ 1$ with gene X & $\circ 9$ with gene X \\
$\circ 9$ without gene X & $\circ 1$ without gene X \\
50 who didn't smoke in their twenties & 2 who didn`t smoke in their twenties \\
$\circ$ all without gene X & $\circ$ all without gene X
\end{tabular}

How likely is the following conditional?

(9) If Jones smoked in his twenties, he has gene X.

Jones most likely doesn't have cancer. Supposing he doesn't, then he most likely doesn't have gene $\mathbf{X}$ if he smoked in his twenties. So, intuitively, the likelihood of (9) is low. However, were we to learn that Jones smoked in his twenties, this would raise the probability we assign to his having cancer (since smoking is a reliable cause of cancer), and thus we would think it likely that he has gene X. Thus, (9) is a Ramsey-violating conditional in Smoking. This is unsurprising - the numbers are exactly the same as in Hospital. However, in this case, Kaufmann's theory predicts that (9) is Ramsey-violating only if its probability depends on the background chance variable $\mathbf{C}$ (whether Jones has cancer). But it is stipulated in Smoking that whether Jones has cancer causally depends on whether he smoked in his twenties. So, Kaufmann's causal independence condition 2 seems to be wrong. ${ }^{13}$

Finally, I have a worry about the proposal that conditionals be evaluated relative to objective-chance-determining variables. Kaufmann argues that the interpretation of the indicative conditional $\ulcorner$ if $\mathrm{A}, \mathrm{B}\urcorner$ at $\bar{A}$-worlds goes by the interpretation of the corresponding subjunctive conditional at those worlds (see especially [kaufmann:2005b], pp. 205-206, 214-215; [kaufmann:2009a], p. 36). If this were correct, and if subjunctive conditionals bore the intimate relationship to objective chances that they are normally thought to have (cf. [lewis:1981c, edgington:1995]), this would be a plausible argument that we interpret indicative conditionals relative to an objective-chance-determining variable. However, indicative and subjunctive conditionals do not generally pattern in the way Kaufmann supposes, as shown by the non-equivalence of the famous minimal pairs due to Ernest Adams

\footnotetext{
${ }^{13}$ Another option would be to deny Kaufmann's theory of local interpretations and try to generate the low probability for (9) some other way (without appealing to partition dependence). I take it this is not a friendly move for Kaufmann to make. I will also not be adopting this strategy.
} 
([adams:1970]):

(10) a. If Oswald didn't shoot Kennedy, then someone other than Oswald shot Kennedy.

b. If Oswald hadn't shot Kennedy, then someone other than Oswald would have shot Kennedy.

Let ' $\bar{O} \rightarrow_{c} S$ ' denote the proposition expressed by the indicative (10-a) and ' $\bar{O} \square \rightarrow_{c} S$ ' denote the proposition expressed by the subjunctive (10-b) in context $c$. In general, we don't want $P\left(\bar{O} \rightarrow_{c} S \mid O\right)=P\left(\bar{O} \square \rightarrow_{c} S \mid O\right)$. The latter value is intuitively low (because we think there was no backup shooter in place), but the former value must be high, given that $P\left(\bar{O} \rightarrow_{c} S\right)=P(S \mid \bar{O})$ and that both values are high (since we're almost certain Kennedy was shot). ${ }^{14}$

I want to stress that none of these challenges constitute knockdown objections to Kaufmann's theory. The first three are just observations that the theory does not yet answer questions (a)-(c) (questions, I should note, that Kaufmann was not aiming to answer with his theory), the fourth challenges a proposed constraint on how conditionals are interpreted relative to partitions, and the fifth challenges a possible motivation for thinking conditionals are interpreted relative to partitions. Nonetheless, since Kaufmann's proposal that the probabilities of conditionals are partition-relative is such a promising account of the failures of RAMSEY'S OBSERVATION, I think these challenges motivate looking for new ways of implementing a partition-relative semantics for conditionals, one which hopefully can answer our questions (a)-(d). In the rest of this paper, I will argue for such a theory.

\section{What's needed to predict the counterexamples}

In order to regiment our discussion, let's introduce some formalism to bridge the sentence/proposition divide. I'll use uppercase roman letters $(\mathrm{A}, \mathrm{B}, \mathrm{C}, \ldots)$ to denote sentences and uppercase italics $(A, B, C, \ldots)$ to denote the propositions expressed by those

\footnotetext{
${ }^{14}$ Perhaps Kaufmann only intended his argument to apply to future-directed indicative conditionals like (i) rather than past indicatives like (10-a).

(i) If Oswald doesn't shoot Kennedy, then someone other than Oswald will shoot Kennedy.

If so, then his claim would be much more plausible, since as [edgington:1995, edgington:2004] has argued, future indicatives (like (i)) seem to "stand or fall" with their past subjunctive counterparts (in this case, (10-b)). See also [barker:1998, bennett:2003]. However, if this is all Kaufmann aims to claim, then his account is incomplete since it won't extend to past indicative exceptions to RAMSEY'S OBSERVATION like $(3)$.
} 
sentences (in the relevant contexts). I will use corner quote notation to talk about the class of indicative conditionals generally: thus, the substitution instances of $\ulcorner$ if $\mathrm{A}, \mathrm{B}\urcorner$ are all and only the indicative conditionals of English. Let $A \rightarrow_{c} B$ denote the proposition expressed by $\ulcorner$ if $\mathrm{A}, \mathrm{B}\urcorner$ as uttered in $c .{ }^{15}$

We begin by defining the following two properties: ${ }^{16}$

- A conditional $\ulcorner$ if $\mathrm{A}, \mathrm{B}\urcorner$ is antecedent independent in $c$ iff $P\left(A \rightarrow_{c} B\right)=P\left(A \rightarrow_{c}\right.$ $B \mid A)$.

- A conditional $\ulcorner$ if $\mathrm{A}, \mathrm{B}\urcorner$ is probability centered in $c$ iff $P\left(A \rightarrow_{c} B \mid A\right)=P(B \mid A)$.

Antecedent independence means roughly that learning the antecedent does not affect the probability of the conditional. Probability centering means roughly that learning the antecedent of a conditional collapses its probability with the probability of its consequentthus, the probability one would have in $A \rightarrow B$ were one to learn $A$ just is the probability one would have in $B$ were one to learn with certainty that $A$ (and nothing else). Notice that any conditional $\ulcorner$ if $\mathrm{A}, \mathrm{B}\urcorner$ that is both probability centered and antecedent independent will be Ramsey-friendly: ${ }^{17}$

- A conditional $\ulcorner$ if $\mathrm{A}, \mathrm{B}\urcorner$ is Ramsey-friendly in $c$ iff $P\left(A \rightarrow_{c} B\right)=P(B \mid A)$.

Therefore, by contraposition, any Ramsey-violating conditional in $c$ must be such that either it is not probability centered in $c$ or is not antecedent independent in $c$. Hence, since (3) is Ramsey-violating in the context Hospital, it must either fail to be probability centered or antecedent independent in that context. Intuitively, your prior probability of (3) is low, since you antecedently think it more likely that Jones is from Appleton than Zephyr, and most people from Appleton that show symptoms of B-flu do not in fact have it. But the probability of (3) given that Jones is showing symptoms of B-flu is high-this is because learning that Jones is showing symptoms of B-flu makes it more likely that he's from Zephyr than Appleton, and hence more likely that showing symptoms of B-flu is indicative of having B-flu. This suggests the following promising approach to diagnosing the failures

\footnotetext{
${ }^{15}$ The assumption that indicative conditionals express propositions is of course controversial. I adopt it here to ease presentation. As we'll see in $\S 6.2$, there are implementations of my favored semantic theory which are not propositional (in the sense of having as their semantic contents something that determines a set of possible worlds).

${ }^{16}$ My thinking in this section was inspired by [rothschild:2013a]'s excellent discussion.

${ }^{17}$ This observation has been made by [lewis:1976, ellis:1978, rothschild:2013a].
} 
of RAMSEY'S OBSERVATION: indicative conditionals are always probability centered, and antecedent independence typically holds, except for certain conditionals in certain contexts (like (3) in Hospital).

Furthermore, there is good reason to believe that probability centering is a universal property of all indicatives in all contexts. Notice that this fact follows from the fact that indicative conditionals are strongly centered in every context, which is independently quite plausible:

- A conditional $\ulcorner$ if $\mathrm{A}, \mathrm{B}\urcorner$ is strongly centered in $c$ iff $\models A \supset\left(B \equiv A \rightarrow_{c} B\right)$.

Suppose that every indicative conditional is strongly centered in every context. Then pick an arbitrary conditional $\ulcorner$ if $\mathrm{A}, \mathrm{B}\urcorner$ and context $c$. Since $\ulcorner$ if $\mathrm{A}, \mathrm{B}\urcorner$ is strongly centered in $c$, $A \rightarrow_{c} B$ and $B$ are true at exactly the same $A$-worlds. Hence, $P\left(A \rightarrow_{c} B \mid A\right)=P(B \mid A)$. Therefore, $\ulcorner$ if $\mathrm{A}, \mathrm{B}\urcorner$ is probability centered in $c$. But since the choice of conditional and context were arbitrary, this holds for every conditional in every context.

Therefore, evidence supporting the claim that every conditional is strongly centered in every context is evidence supporting the claim that all conditionals are probability centered in all contexts. Here is some evidence supporting strong centering. Suppose we place a bet on the outcome of John's recent die roll (the outcome of which remains unknown). I bet that if John rolled a prime then he rolled an odd and you bet against me. It seems that if John rolled an odd prime then I win the bet, while if John rolled an even prime, you win the bet (set aside what happens if John rolls a non-prime for now). If our betting intuitions are evidence for the truth conditions of the conditional, then it seems that $A B$ is sufficient for the truth of $A \rightarrow_{c} B$, while $A \bar{B}$ is sufficient for the falsity of $A \rightarrow_{c} B$. Furthermore, the choice of context and conditional here are arbitrary. Therefore, our betting intuitions support the claim that every conditional is strongly centered in every context. ${ }^{18}$

\footnotetext{
${ }^{18}$ One might think instead that $A \rightarrow_{c} B$ is false if it is epistemically possible that $A \bar{B}$, since it is odd to utter a conditional if it's epistemically possible that its antecedent is true and consequent false. However, as [moss:2012] points out, this thought is at odds with how such conditionals embed (using (2) as our example):

(2) If John rolled a prime number, then he rolled an odd number.

(i) a. It's probably the case that if John rolled a prime number, then he rolled an odd number.

b. \#It's not the case that if John rolled rolled a prime number, then he rolled an odd number.

Now, if (2) were false (and indeed, this were knowable merely by reflection on the open possibilities in the context), we'd have no explanation of why (i-b) is infelicitous. On the other hand, if (2) were not false (as
} 
I will now take it for granted that a plausible strategy for accounting for the exceptions to RAMSEY'S OBSERVATION will be a semantics for conditionals that predicts that they are probability centered in every context and antecedent independent in most, but not all, contexts. Given this starting point, we can now prove the following theorem (proof in Appendix):

Theorem 1. $\ulcorner$ if $\mathrm{A}, \mathrm{B}\urcorner$ is Ramsey-friendly in $c$ iff $P\left(A \rightarrow_{c} B \mid \bar{A}\right)=P(B \mid A)$.

This theorem confirms a feature of indicative conditionals revealed by the Oracle example, which is that the probability of $A \rightarrow_{c} B$ depends on more than simply the behavior of $B$ throughout the $A$-region of logical space. Indeed, Theorem 1 tells us that if we are to predict conditionals which are exceptions to RAMSEY'S OBSERVATIONS, they will be ones whose probability differs across the antecedent/non-antecedent region of the space of epistemically possible worlds (epistemic space). This is exactly what theories which take RAMSEY'S OBSERVATION as their starting point cannot predict (e.g., [adams:1975, edgington:1995, bennett:2003]). Therefore, to find a semantics for conditionals that can predict exceptions to RAMSEY'S OBSERVATION, we should look for a semantics of conditionals which ensures that the probability of a conditional given the negation of its antecedent is both (i) non-trivial (that is, some value between 0 and 1) and (ii) counts towards the probability of the conditional as a whole. Graphically, this means that the probability of a conditional should extend somewhat into the non-antecedent region of epistemic space, but not fill it completely, as in Figure 1:

I allege), we have a natural explanation of why (i-b) is unassertable - it is false.

Furthermore, there is a plausible alternative explanation for the oddness of uttering a conditional if it's epistemically possible that its antecedent is true and consequent false, which is that in such a case, it would be epistemically possible that the conditional is false. And in that case, the usual kinds of norms of assertion (knowledge, or being justified in believing) would not be satisfied. See [khoo:2013c] for further discussion. 


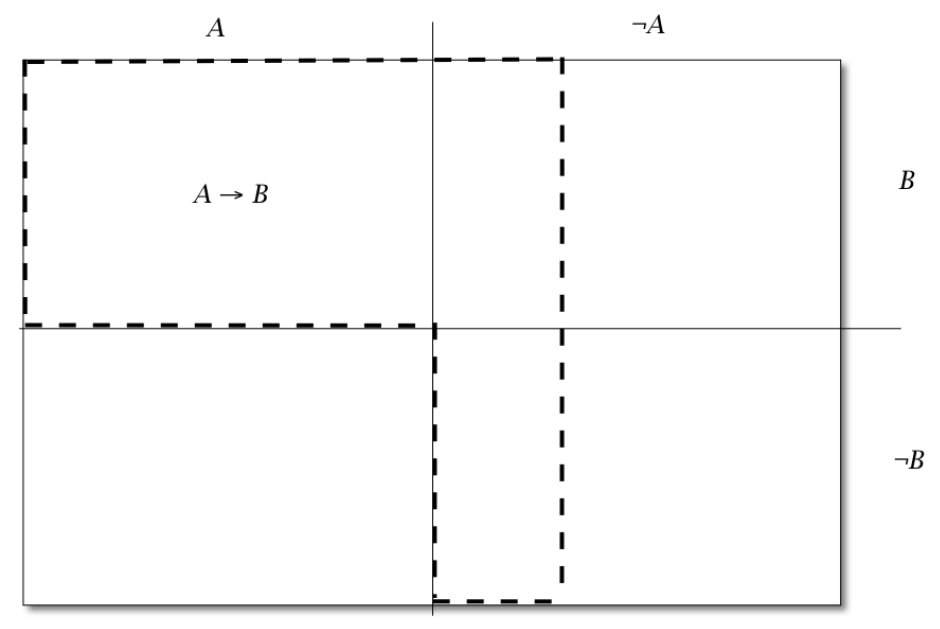

Figure 1

In $\S 4$, I provide such a semantics for conditionals, which will yield my answer to (a). Then, in $\S 5$, I show how we can augment this semantics with a plausible pragmatics, which I argue delivers compelling answers to (b)-(d).

\section{Incorporating partition dependence into the semantics}

My aim in this section is to motivate a uniform semantics for indicative conditionals that can generate Ramsey-violating and Ramsey-friendly interpretations of conditionals. As we saw in the previous section, to do so, we need the probability of an indicative conditional given the negation of its antecedent to non-trivially count towards the probability of the conditional as a whole, which means that the conditional must be true at some, but not all, non-antecedent worlds. Unfortunately, this is an awkward demand to fulfill, as we seem to lack clear intuitions about the truth values of indicative conditionals evaluated at worlds where their antecedents are false. For instance, it is not clear to me whether (3) would be true or would be false at an epistemically possible world $w$ where Jones isn't showing symptoms of B-flu. ${ }^{19}$

\footnotetext{
${ }^{19}$ Remember, I argued in footnote 18 that (3) isn't automatically false simply because it's epistemically possible that Jones is showing symptoms of B-flu even though he doesn't have it, and I argued in $\S 2$ that whether (3) is true at such a world isn't just a matter of its corresponding subjunctive conditional (i) being true there:
}

If Jones had been showing symptoms of B-flu, he would have had B-flu. 
If Jones is showing symptoms of B-flu, he has B-flu.

Even worse, it is not clear what I could learn about this world that would rationally persuade me to think that (3) is true there, or false there. At this point, I think the best option is to conclude that it is indeterminate whether (3) is true or false at $w-$ remaining neutral for now as to the nature of this indeterminacy. ${ }^{20}$ Given this observation, we might despair, for how are we to generate a predictive theory about the probabilities of conditionals on which they have non-trivial probabilities at the region of epistemic space where their antecedents are false if we don't have clear intuitions about their truth values at the worlds in that region?

Nonetheless, there is a strategy for determining indirectly the conditional's truth value at worlds where its antecedent is false, given our intuitions about its overall probability. Compare (3) with the following:

If Jones flipped the coin, it landed heads.

Suppose we know that the coin is double-headed but not whether Jones flipped it. Knowing this, we should be certain that (11) is true. But then given that the probability of (11) at the region of epistemic space where its antecedent is false counts towards its overall probability, (11) must be certainly true given that Jones didn't flip the coin. That means that (11) must be true at all of the epistemically possible worlds where Jones didn't flip the coin! Therefore, we have a partial indirect strategy for determining whether a conditional is true or false at epistemically possible worlds where its antecedent is false: if we are certain of $B$ given $A$, then we should be certain that $\ulcorner$ if $\mathrm{A}, \mathrm{B}\urcorner$ is true, and hence should think that $\ulcorner$ if $\mathrm{A}, \mathrm{B}\urcorner$ is true at each epistemically possible $\bar{A}$-world.

Robert Stalnaker's influential theory of indicative conditionals predicts these features of indicative conditionals (see [stalnaker:1968, stalnaker:1970, stalnaker:1975, stalnaker:1980]). As such, I will take it as the starting point for my theory. Roughly, Stalnaker's theory says that a conditional $\ulcorner$ if $\mathrm{A}, \mathrm{B}\urcorner$ is true at a world just if $B$ is true at the closest epistemically possible $A$-world to it. A bit more formally:

\footnotetext{
${ }^{20}$ For instance, the indeterminacy may be semantic (due to multiple equally good interpretations of our language) and hence that the conditional is neither true nor false at such worlds. Or it may be epistemic (owing to a kind of in principle ignorance of the workings of our language), and the conditional is either true or false at such worlds, but we simply don't know which. The indeterminacy may even be metaphysicalsuch worlds may themselves be indeterminate as to whether the conditional is true or false at them.
} 


\section{Stalnaker semantics:}

$\ulcorner$ if $\mathrm{A}, \mathrm{B}\urcorner$ is true at $c, w$ iff $\mathrm{B}$ is true at $c, w^{\prime}$; where $w^{\prime}$ is the $c$-closest epistemically $\operatorname{accessible}_{c} A$-world to $w$

I suppose that a context $c$ determines $E_{c}$, the set of epistemically accessible ${ }_{c}$ worlds, $P_{c}$, a unique probability function that is normalized on $E_{c}$ (i.e., $P_{c}\left(E_{c}\right)=1$ ), and a set of admissible $A$-closeness functions for that context (which determine, in some as yet unspecified way, the $c$-closest world to each world in $\left.E_{c}\right){ }^{21}$ We round out the semantics with two final assumptions: (i) that $\ulcorner$ if $\mathrm{A}, \mathrm{B}\urcorner$ is defined at $w, c$ only if $w \in E_{c}$ and $A$ is compatible with $E_{c}$, (ii) that the set of admissible $A$-closeness functions is the largest set of functions from worlds to worlds containing only functions defined on worlds in $E_{c}$ such that each maps every world in $E_{c}$ to a unique $A$-world in $E_{c}$, and maps each $A$-world in $E_{c}$ to itself. Together, these two assumptions yield:

Centering: if $A$ is true at $w$, then the $c$-closest $A$-world to $w$ is $w$.

E-constraint: the $c$-closest $A$-world to $w$ is also within $E_{c}$.

Centering ensures that Stalnaker semantics predicts that every indicative obeys modus ponens (at least for simple sentences), is strongly centered in every context (and hence probability centered in every context), ${ }^{22}$ and is stronger than its corresponding material conditional. E-constraint ensures that indicative conditionals are epistemic in the sense that the truth value of a conditional should depend only on what happens at epistemically possible worlds (cf. [stalnaker:1975]: 275-6). This predicts, correctly, as we saw above, that if $P(B \mid A)=1$ then $\ulcorner$ if $\mathrm{A}, \mathrm{B}\urcorner$ will be certainly true, given the natural assumption that $\ulcorner$ if $\mathrm{A}, \mathrm{B}\urcorner$ is certainly true in $c$ iff it is true at every world in $E_{c}$ as evaluated with respect to every one of the admissible $A$-closeness functions determined by $c$. In the case at hand, where $P(B \mid A)=1$, $\ulcorner$ if $\mathrm{A}, \mathrm{B}\urcorner$ will be true at every $A$-world in $E_{c}$ (since all are $B$-worlds) and will be true at every $\bar{A}$-world in $E_{c}$ (since every candidate $c$-closest $A$-world to any of them is a $B$-world). ${ }^{23}$

\footnotetext{
${ }^{21}$ I leave off the subscript on $P$ in what follows to ease readability. However, understand my claims throughout as about the probability function determined by the relevant context $c$, even if this relativization is not made explicit.

${ }^{22}$ Let $\ulcorner$ if $\mathrm{A}, \mathrm{B}\urcorner$ be an arbitrary conditional and $c$ an arbitrary context. Given Stalnaker semantics and Centering, $A \rightarrow_{c} B$ and $B$ are true at exactly the same $A$-worlds in $E_{c}$. Hence, $P\left(A \rightarrow_{c} B \mid A\right)=P(B \mid A)$, and thus $\ulcorner$ if $\mathrm{A}, \mathrm{B}\urcorner$ is probability centered in $c$.

${ }^{23}$ This assumes the domain is finite; in the infinite case, it may be the case that $P(B \mid A)=1$ even though there is a non-empty set of $A$-worlds in which $B$ is false.
} 
However, notice that Stalnaker's two constraints on $c$-closeness often do not ensure that there will be a unique $c$-closest $A$-world to each world in $E_{c}$ (they do iff there is only one $A$-world in $E_{c}$; in that case there will be only one way of mapping $\bar{A}$-worlds in $E_{c}$ to $A$-worlds in $E_{c}$, namely, map them all to the single $A$-world in $\left.E_{c}\right)$. In contexts where there is not a unique $c$-closest $A$-world to each world in $E_{c}$ and there are epistemically accessible $A B$-worlds and $A \bar{B}$-worlds, Stalnaker's semantics predicts that $\ulcorner$ if $\mathrm{A}, \mathrm{B}\urcorner$ will be indeterminate in truth value at every epistemically possible $\bar{A}$-world (since there will be multiple candidate $A$-closest worlds for each $\bar{A}$-world in $E_{c}$, some $B$-worlds and some $\bar{B}$-worlds). As we saw with our intuitions about (3) above, this seems to be just the right result (remaining neutral for now about the nature of this indeterminacy, and also setting aside for now how to draw on this result to predict the right probabilities of conditionals).

I turn now to motivate a generalization of Stalnaker's E-constraint. To begin, consider the following argument for why (4-a) is likely true in Oracle:

a. If the oracle is correct, the coin landed heads.

Either the oracle said "heads" or she said "tails" (though most likely she said "heads"). Suppose we were to learn she said "heads"; in that case we should be certain that (4-a) is true, since every world where the oracle is correct and said "heads" is a world where the coin landed heads. But suppose instead we were to learn that she said "tails"; in that case we should be certain that (4-a) is false, since every world where the oracle is correct and said "tails" is a world where the coin landed tails. But then the overall probability that (4-a) is true should equal the likelihood that the oracle said "heads". Since it's likely that the oracle said "heads," (4-a) is likely. ${ }^{24}$

I submit that this is an intuitively compelling piece of reasoning. But Stalnaker semantics + Centering + E-constraint predicts that the inference from:

\footnotetext{
${ }^{24}$ Analogous reasoning can be given to show that (4-b) is unlikely, when we draw on the partition whether the coin landed heads or not (the latter of which is more likely, since the coin was weighted towards tails):

(4) b. If the oracle is correct, she said "heads".

Suppose the coin landed heads; in that case we should be certain that (4-b) is true, since every world where the oracle is correct and the coin landed heads is one in which she said "heads". Suppose instead the coin landed tails; in that case we should be certain that (4-b) is false, since every world where the oracle is correct and the coin landed tails is one in which she said "tails". So, the overall probability that (4-b) is true should equal the likelihood that the coin landed heads. But this is very unlikely (since the coin was weighted towards tails). So (4-b) is unlikely.
} 
(a) Every world in $E_{c}$ where the oracle is correct and said "heads" is a world where the coin landed heads.

to

(b) (4-a) is certainly true, given that the oracle said "heads".

is invalid as long as there are some worlds in $E_{c}$ where the oracle is correct and the coin landed tails (as is the case in Oracle). To see why the theory makes this prediction, remember that we are assuming that $\ulcorner$ if $\mathrm{A}, \mathrm{B}\urcorner$ is certainly true in $c$ iff it is true at every world in $E_{c}$, as evaluated with respect to each of the admissible $A$-closeness functions determined by $c$ - this is necessary for the semantics to predict that one is certain that $B$ given $A$ iff one is certain that $\ulcorner$ if $\mathrm{A}, \mathrm{B}\urcorner$ is true. Given this assumption, (b) holds only if every admissible $A$-closeness function maps every world where the oracle said "heads" to a world where the oracle is correct and the coin landed heads. But given that the only constraints on admissible $A$-closeness functions are Centering and E-constraint, there will be an admissible $A$-closeness function that maps some said- "heads"-worlds to a world where the oracle is correct and the coin lands tails. This is depicted in Figure 2, where $w_{4}$ is mapped to $w_{2}$ via the closeness function represented by the hashed line:

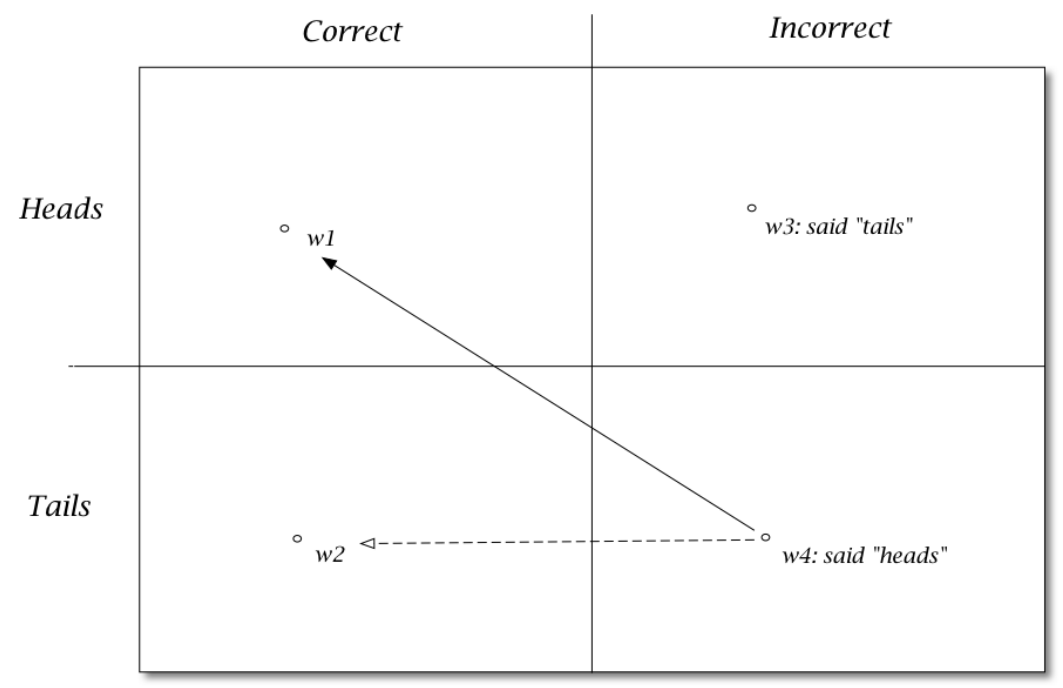

Figure 2 
In such a world (in this case, $w_{4}$ ), evaluated with respect to that admissible $A$-closeness function (represented by the hashed line), (4-a) is false. As such, the theory predicts that (4-a) will not be certainly true, given that the oracle said "heads", and thus (b) is false. Furthermore, all this is true even if (a) is true. Thus, Stalnaker semantics + Centering + E-constraint predicts that the inference from (a) to (b) is invalid.

This is an unfortunate result, since the reasoning above seems so compelling (indeed, it seems to underlie the intuition that (4-a) is likely true). I propose the following solution: conditionals are interpreted relative to a salient partition on $E_{c}$ which constrains the set of admissible $A$-closeness functions to contain only those functions that map each world $w$ to some $A$-world which falls in the same cell of the salient partition as $w{ }^{25}$ Notice that this is just a generalization of Stalnaker's E-constraint - it ensures that the truth value of a conditional at worlds in each cell of the salient partition depends only on what happens at other worlds in that cell. To state this generalization formally, let $\mathbf{Z}_{c}$ be the partition that $\ulcorner$ if $\mathrm{A}, \mathrm{B}\urcorner$ is interpreted relative to, and let $[w] \mathbf{Z}_{c}$ be the cell of $\mathbf{Z}_{c}$ that $w$ falls within. Here is the generalization of E-constraint that I propose: ${ }^{26}$

Serious partitioning: where $\mathbf{Z}_{c}$ is the partition $c$ assigns to $\ulcorner$ if $A, B\urcorner$ : the $c$-closest $A$-world to $w$ is among $[w] \mathbf{Z}_{c}$.

I also add the following two definedness constraints on $\ulcorner$ if $\mathrm{A}, \mathrm{B}\urcorner$ :

$\ulcorner$ if $\mathrm{A}, \mathrm{B}\urcorner$ is defined at $c, w$ only if

A-compatibility: $[w] \mathbf{z}_{c} \cap A \neq \emptyset$, and

Accessibility: $w \in E_{c}$.

A-compatibility is motivated by how the semantics is supposed to work. When evaluating whether $A \rightarrow_{c} B$ holds at some $\bar{A}$-world $w$, the semantics looks for the $c$-closest $A$-world to $w$. But given Serious partitioning, if $[w] \mathbf{z}_{c} \cap A=\emptyset$, then no worlds will be candidates for being the $c$-closest $A$-world to $w$ and the semantics will crash. Thus, the A-compatibility definedness constraint follows directly from combining Stalnaker semantics with Serious

\footnotetext{
${ }^{25}$ [starr:2014b, moss:2015] also integrate partition-sensitivity into their semantics for conditionals, but in different ways than I do here.

${ }^{26}$ This is a generalization of E-constraint because, in the limiting case where the conditional is interpreted relative to the trivial partition $\left\{E_{c}\right\}$, this constraint makes the same demand on $c$-closeness as E-constraint.
} 
partitioning. Next, Accessibility ensures that $A \rightarrow_{c} B$ is undefined at $w$ if $w$ is not epistemically accessible given the information in $c$. This constraint is motivated by the observation that indicative conditionals are odd and hard to hear as either true or false in a context in which it's taken for granted that their antecedents are false, and hence in which there are no $A$-worlds in $E_{c}$ (cf. [warmbrod:1983, edgington:1991, bennett:2003]): ${ }^{27}$

It's definitely not raining. \#If it is raining, there are clouds in the sky.

Call the resulting theory, which combines Stalnaker semantics, Centering, and Serious partitioning, The SP theory. As with the basic Stalnakerian theory, The SP theory predicts that indicative conditionals obey modus ponens, are strongly centered, and express propositions stronger than material conditionals (see Appendix for proofs). However, unlike the basic Stalnakerian theory, The SP theory predicts that the reasoning about the probabilities of (4-a)/(4-b) discussed above is sound, as long as they are interpreted relative to the right partitions $\left(\mathbf{S}_{\mathbf{H}}\right.$ for (4-a) and $\mathbf{H}$ for (4-b)). As we'll see in $\S 5$, when combined with an independently plausible theory about what sets the partition relative to which a conditional is interpreted, The SP theory will also make the right predictions about the various Ramsey-friendly and Ramsey-violating conditionals we saw in $§ 1 .^{28,29}$

Recall the key feature distinguishing Ramsey-friendly from Ramsey-violating conditionals from $\S 3$ - $\ulcorner$ if $\mathrm{A}, \mathrm{B}\urcorner$ is Ramsey-friendly in $c$ iff $P\left(A \rightarrow_{c} B \mid \bar{A}\right)=P(B \mid A)$ and is

\footnotetext{
${ }^{27}$ Of course, it is possible to hear strings like (12) as felicitous, but only if it's understood that the speaker is elaborating her reasons for accepting the first sentence-for instance using the conditional to perform an implicit modus tollens on "it is raining". In those cases, the conditional is most naturally interpreted relative to a different set of worlds than the one "it is definitely not raining" is true relative to.

${ }^{28}$ I've assumed that the intuitively compelling reasoning about (4-a) above is sound and that it is a strength of a theory that it predicts as much. However, people who think the McGee/Kaufmann counterexamples to RAMSEY'S OBSERVATION involve cognitively tempting mistakes would probably also regard this reasoning as invalid, even if some of us find it compelling. A full defense of the claim that the Ramseyviolating intuitions are correct goes beyond the scope of this paper; however, I do think that one (small) point in favor of thinking such intuitions are correct is the fact that an elegant minimal extension of an independently compelling theory predicts that they are (since we can at least see what might be responsible for them). In what follows, I will argue that The SP theory, plus my pragmatics in $\S 5$, is such a theory.

${ }^{29}$ An anonymous reviewer prompted me to think about whether The SP theory predicts that merely varying the partition some conditional is evaluated relative to will change the truth value the conditional is predicted to have at some possible world. First, notice that, on the theory, $\ulcorner$ if $\mathrm{A}, \mathrm{B}\urcorner$ is true (in $c$ ) at any $A B$-world and false at any $A \bar{B}$-world in $E_{c}$, regardless of the partition the conditional is evaluated relative to. However, the theory predicts that the truth value of $\ulcorner$ if $\mathrm{A}, \mathrm{B}\urcorner$ at some $\bar{A}$-world may depend on the partition it is evaluated relative to. In the Appendix, I show how we can determine this indirectly in certain abstract cases. It turns out that the kinds of cases in which this may happen are similar to "standoff" cases ([gibbard:1981, edgington:1995, bennett:2003]). I sketch an example of such a case in the Appendix and argue that The SP theory's prediction in that case is actually quite plausible.
} 
Ramsey-violating iff $P\left(A \rightarrow_{c} B \mid \bar{A}\right) \neq P(B \mid A)$. Since according to The SP theory, the choice of background partition may affect $P\left(A \rightarrow_{c} B \mid \bar{A}\right)$, the theory is well-placed to explain then why conditionals interpreted relative to certain background partitions are Ramsey-violating. But in order for it to do so, the theory must predict the right value for $P\left(A \rightarrow_{c} B \mid \bar{A}\right)$; how should it do so? First off, the probability of $A \rightarrow_{c} B$ given $\bar{A}$ should equal the expectation that the $c$-closest $A$-world to any $\bar{A}$-world will be a $B$-world. Exactly how we calculate this expectation will depend on how we think about the indeterminacy that arises when there is more than one admissible $A$-closeness function in $c$. Suppose for now that $P_{c}$ is uniform - that we invest equal confidence in each world that it is actual. Then, if each admissible $A$-closeness function is as likely as any other to deliver the $c$-closest $A$-world to any world in $E_{c}$, for each $Z \in \mathbf{Z}_{c}$, our expectation that the $c$-closest $A$-world to any $\bar{A} Z$-world will be a $B$-world should equal the probability of $B$ given $A Z$. But then distributing the probability of $A \rightarrow_{c} B$ given $\bar{A}$ across cells in $\mathbf{Z}_{c}$ yields:

$$
\text { Prob: } P\left(A \rightarrow_{c} B \mid \bar{A}\right)=\sum_{Z \in \mathbf{Z}_{c}} P(B \mid Z A) \cdot P(Z \mid \bar{A})
$$

Thus, we have an informal derivation of what we expect The SP theory to predict about the probability of conditionals given the negation of their antecedents. A rigorous derivation of Prob requires taking a stand on the kind of indeterminacy (semantic, epistemic, metaphysical) that arises given that Serious partitioning constrains without determining a unique closeness relation in the context. We'll return to this point in $\S 6$, where I show how two different implementations of The SP theory entail (their own versions of) Prob. For now, I close by noting that, given Prob and Centering, The SP theory entails the following (see the Appendix for proofs):

Theorem 2. $P\left(A \rightarrow_{c} B\right)=P\left(A \rightarrow_{\ell}^{\mathbf{Z}_{c}} B\right)$; where $\mathbf{Z}_{c}$ is the partition assigned to $A \rightarrow{ }_{c} B$.

Theorem 3. For any conditional $\ulcorner$ if $\mathrm{A}, \mathrm{B}\urcorner$ and context $c$, if the partition for $\ulcorner$ if $\mathrm{A}$, $\mathrm{B}\urcorner$ in $c$ is $\left\{E_{c}\right\}$, then $\ulcorner$ if $\mathrm{A}, \mathrm{B}\urcorner$ is Ramsey-friendly in $c$.

Theorem 2 states that the probability of $A \rightarrow_{c} B$ is equal to the probability of Kaufmann's "local" interpretation of the conditional whenever they share the same background partition. Theorem 3 states that a conditional is Ramsey-friendly in a context where it is assigned the trivial partition in that context. (In $\S 5.3 .3$, I'll appeal to another result to 
articulate the theory's explanation of why Ramsey-violating conditionals are rare.) These results pave the way to a unified account of Ramsey-friendly and Ramsey-violating interpretations of conditionals - on this theory, all conditionals receive "local" (in Kaufmann's sense) interpretations and the Ramseyan variability is explained by facts about which partition they are interpreted relative to in different contexts (this is my theory's answer to (a)). Taking for granted that there is a plausible implementation of The SP theory that entails Prob, my goal in the next section is to motivate a suitable pragmatic theory of the relevant background partition to combine with The SP theory so that it generates reasonable answers to our remaining questions (b)-(d) from $\S 1$.

\section{Ramseyan variations}

Recall the questions raised in $\S 1$ :

a. What is it about the semantics of conditionals in virtue of which they give rise to Ramsey-violating and Ramsey-friendly interpretations?

b. Why do certain conditionals favor Ramsey-violating "local" interpretations in certain contexts?

c. Why, in such contexts, can we sometimes override the Ramsey-violating interpretations in favor of Ramsey-friendly interpretations?

d. Why are Ramsey-violating interpretations of conditionals so rare?

In this section, I will motivate a pragmatic theory of what sets a conditional's partition in context. I'll argue that combining this pragmatics with The SP theory yields a theory which provides plausible answers to (b)-(d). Here's a quick summary of the answers my theory will deliver. Regarding (b), my theory predicts that the default interpretation of a conditional assigns it the most salient admissible partition, and that in a context in which it has a Ramsey-violating interpretation, it is interpreted relative to a salient question under discussion. Regarding (c), I'll argue that the default interpretation may be overridden if the speaker makes clear her intention that the conditional she utters receive a non-default interpretation, and this is what gives rise to Ramsey-friendly interpretations in contexts that would normally favor a Ramsey-violating interpretation. Finally, regarding (d), I'll 
show why the theory predicts that the conditions under which a conditional can get a Ramsey-violating interpretation are rare.

I ultimately want to remain neutral about exactly what features of the discourse context fix the partition relative to which some conditional is interpreted. ${ }^{30}$ Nonetheless, I can still draw on general pragmatic principles to motivate the following constraint on which partition will be assigned to some conditional in some context:

Admissibility: $\ulcorner$ if $\mathrm{A}, \mathrm{B}\urcorner$ will be interpreted in $c$ relative to $\mathbf{Z}$ only if $\mathbf{Z}$ is an admissible partition for $\ulcorner$ if $\mathrm{A}, \mathrm{B}\urcorner$ in $c$.

- Where $\mathbf{Z}$ is an admissible partition for $\ulcorner$ if $\mathrm{A}, \mathrm{B}\urcorner$ in $c$ iff

1. $\ulcorner$ if $\mathrm{A}, \mathrm{B}\urcorner$ is well defined in $c$ relative to $\mathbf{Z}$,

2. $\ulcorner$ if $\mathrm{A}, \mathrm{B}\urcorner$ is not trivial in $c$ relative to $\mathbf{Z}$, and

as well as the following default interpretation for indicative conditionals:

Default partition: Generally (given no other countervailing interpretive considerations), $\ulcorner$ if $\mathrm{A}, \mathrm{B}\urcorner$ will be interpreted in $c$ relative to the most salient admissible partition for $\ulcorner$ if $\mathrm{A}, \mathrm{B}\urcorner$ in $c$.

Before proceeding, I want to emphasize that Default partition is a default hypothesis which may be subject to exceptions if countervailing interpretive considerations are salient in the context. In particular, as we'll see in $§ 5.2$, one such countervailing interpretive consideration will be a speaker making clear her intention that some conditional receive a non-default interpretation. Furthermore, although I will not here argue for an exhaustive list of interpretative considerations that may override Default partition, I don't think this makes my theory vacuous. In particular, by seeing what motivates Default partition, we have some sense of the sort of circumstances it may fail-these ought to be ones in which the considerations that motivate it are overridden. To that extent, my proposal here should be taken as a reasonable, independently motivated, first attempt at a pragmatic theory to combine with my semantics for conditionals. As with any pragmatic theory, it would be unreasonable to demand that it generate correct predictions about any possible conditional in any possible context. My goal is an independently motivated theory that

\footnotetext{
${ }^{30}$ In particular, I want to remain neutral about whether the resolution of this contextual parameter is determined by speaker intentions (cf. [perry:2009, king:2013a, king:2013b] on demonstratives or [dowell:2011] on epistemic modal bases), or something more indirect (as in [glanzberg:2007]).
} 
answers questions (b)-(d); I will be satisfied if I can articulate a partial theory that offers compelling answers to those questions. I leave it to further work sorting out the extent to which the theory generalizes to other cases.

In $\S 5.1, I$ will motivate Default partition and Admissibility. Then, in §5.2-5.3, I will show how combining my semantics with this pragmatics provides compelling answers to $(\mathrm{b})-(\mathrm{d})$.

\subsection{Motivating Default partition}

My semantics holds that conditionals are partition-relative. Thus, it is a natural thought that they will be interpreted relative to partitions on the space of epistemic possibilities that are salient in the context. But what makes a partition salient? The most obvious way for an epistemic partition to be salient is if it is a question under discussion in the context. The notion of a question under discussion (QUD) comes from [roberts:1996b, roberts:2012b, roberts:2012a], who argues that we should think of contexts as determining both a set of epistemically accessible worlds ( $E_{c}$ in my system), as well as a stack of questions under discussion in that context, which partition $E_{c} \cdot{ }^{31}$ A question is under discussion in a context if it is a discourse goal to answer it - that is, jointly figure out which cell of it we are in. Roberts draws on this natural idea to provide criteria of relevance for speech acts (relevant speech acts being ones which entail partial answers to, or introduce strategies for answering, questions under discussion). Two important features of Roberts' theory bear emphasizing. The first is that questions are explicitly under discussion if asked in the context, but they may also be implicitly under discussion, as revealed by other speech acts made in the context. The second is that questions under discussion are ordered so that the topmost question in the QUD stack is the question that is immediately under discussion, and hence the one which sets the most pressing discourse goal of the context (which is not to say that answering some other question in the stack would be an irrelevant move, just a less relevant one). As such, we expect the highest question in the QUD stack to the most salient question in the context. ${ }^{32}$

\footnotetext{
${ }^{31}$ For arguments for treating questions as partitions, see [groenendijk:1984, groenendijk:1997].

${ }^{32}$ Thus, we predict that if there are multiple admissible questions under discussion in the context, the conditional will be interpreted relative to the one which is the highest in the stack. I admit that testing this prediction is quite difficult because it is hard to keep the information about how $B$ depends on both $A$ and $\mathbf{Z}$ and $A$ and $\mathbf{X}$ in mind all at once (though see the "partition standoff" case in the Appendix). However, I should point out one prediction of this proposal which does seem plausible, which is that since a sub-question of some question under discussion $Q$ will be higher in the QUD stack than $Q$ (cf.
} 
Now, notice that there is also a natural partition that will be minimally salient in any context - the trivial partition $\left\{E_{c}\right\}$. This partition is always minimally salient because it is "always there" in some sense - it does not require any special work to draw attention to it. Nonetheless, since it is always there, it is boring, and thus it seems natural to assume that questions under discussion will always be more salient than the trivial partition in the context. Finally, I'll assume (provisionally) for now that, besides QUDs and the trivial partition, no other partitions on $E_{c}$ are ever salient in $c$. Let's turn now to the three constraints on admissible partitions for conditionals.

\subsection{Motivating Admissibility}

\section{Well-defined}

In considering whether to accept some sentence as true (as uttered in some context), we usually consider whether the proposition it expresses is sufficiently likely given what we know (or our evidence). But to accurately evaluate the likelihood that a sentence is true requires that the proposition expressed by that sentence be defined at every epistemically accessible world. Thus, if possible, we will aim to interpret any sentence we are considering whether to accept in such a way that the proposition it expresses is defined at every epistemically possible world. Thus, in evaluating whether to accept $\ulcorner$ if $\mathrm{A}, \mathrm{B}\urcorner$ in $c$ as true, we naturally aim to interpret it so that it is defined at each world in $E_{c}$. But recall that according to The SP theory, $\ulcorner$ if $\mathrm{A}, \mathrm{B}\urcorner$ is defined at some $w \in E_{c}$ only if $[w] \mathbf{z}_{c} \cap A \neq \emptyset$. Thus, given the relevant notion of well-defined:

$\ulcorner$ if $\mathrm{A}, \mathrm{B}\urcorner$ is well-defined in $c$ relative to $\mathbf{Z}$ iff $\forall w \in E_{c}:[w] \mathbf{z} \cap A \neq \emptyset$.

it follows that since we aim to interpret $\ulcorner$ if $\mathrm{A}, \mathrm{B}\urcorner$ in $c$ so that $A \rightarrow_{c} B$ is defined at each world in $E_{c}$, we must therefore aim to interpret it relative to a partition on which it is well-defined. ${ }^{33}$ The main upshot of the well-definedness constraint is that we expect a conditional $\ulcorner$ if $\mathrm{A}, \mathrm{B}\urcorner$ not to be interpreted relative to the partition $\{A, \bar{A}\}$ since on this

[roberts:2012a]: 14-15), whenever both questions are admissible partitions for some conditional, it will be interpreted relative to the (strictly finer) sub-question. This provides a way of avoiding the challenge [douven:2008] raises to Kaufmann's theory. Douven sketches an example where two partitions are salient (one which is a sub-partition of the other), and argues that in this case Kaufmann's theory must predict that one conditional has two distinct local probabilities. If my QUD proposal is correct, then the conditional should be interpreted relative to sub-partition (which is the higher QUD in the stack) and thus have only one probability in the context.

${ }^{33}$ Suppose $\ulcorner$ if $\mathrm{A}, \mathrm{B}\urcorner$ is interpreted in $c$ relative to a partition on which it is not well-defined. Then $\exists w \in E_{c}:[w]_{\mathbf{z}} \cap A=\emptyset$. Then there is a $w \in E_{c}$ at which $\ulcorner$ if $\mathrm{A}, \mathrm{B}\urcorner$ is undefined. 
interpretation it would be undefined at all epistemically accessible $\bar{A}$-worlds.

\section{Non-triviality}

Next, notice that we generally interpret a complex sentence so that it is not epistemically equivalent (that is, true/false at all the same epistemically accessible worlds) with one of its constituents. This is motivated by the thought that if someone utters a sentence $S$, which contains $S^{\prime}$ as a constituent, they presumably would have said the simpler sentence if that's all they meant to convey by it - after all, doing so is more efficient and less liable to misinterpretation. As a result, interpretation seems constrained by the following principle:

Avoid trivialization: ceteris paribus, if possible, interpret a complex sentence in $c$ (if possible) so that it is not equivalent throughout $E_{c}$ with any of its sentential constituents.

Here is an example motivating this intuitive principle:

The treasure might be in the attic.

Like conditionals, bare epistemic possibility claims like (14) are interpreted as quantifiers over a set of worlds determined by a contextually-determined modal base $f$, which we can think of as functions from worlds to sets of worlds (cf. [kratzer:1977, kratzer:1981, kratzer:1991]). In particular, (14) expresses the claim that its prejacent (that the treasure is in the attic) holds at some world in the output of its modal base. Kratzer argues that, in principle, modal claims may be interpreted relative to more or less realistic modal bases, with minimally realistic modal bases mapping each world to some set of worlds containing it, and totally realistic modal bases mapping each world to its singleton. Say that the modal base $f$ of some modal settles its prejacent $P$ iff for any world $w, f(w)$ either entails $P$ or entails $\bar{P}$. Thus, in principle, we should expect there to be interpretations of epistemic possibility claims like (14) in which they are epistemically equivalent to their prejacents. ${ }^{34}$ But this almost never happens! ${ }^{35}$ My suggestion is that Avoid trivialization blocks

\footnotetext{
${ }^{34}$ Suppose that $\ulcorner\diamond \mathrm{P}\urcorner$ is interpreted relative to a realistic modal base $f$ that settles $P$. Then at every $P$-world $w, f(w)$ entails $P$, in which case $\ulcorner\diamond \mathrm{P}\urcorner$ is true at $w$, and at every $\bar{P}$-world $w^{\prime}, f\left(w^{\prime}\right)$ entails $\bar{P}$, in which case $\ulcorner\diamond \mathrm{P}\urcorner$ is false at $w^{\prime}$.

${ }^{35}$ Here's a possible context, followed by an explanation why it is in fact evidence for (rather than a challenge to) the point I'm making here. Joe has made a shady deal with one of the organizers of the treasure hunt - they will convey to him the location of the treasure in exchange for half of the prize money. The shady organizer has gathered the treasure hunters to explain the rules of the hunt. In particular, he
} 
us from interpreting epistemic possibility sentences as epistemically equivalent to their prejacents, thus predicting this observation. Though admittedly more work exploring its consequences (and other general theoretical motivations) is needed, I conclude for now that a provisional case has been made in favor of Avoid trivialization.

The primary consequence of Avoid trivialization for the interpretation of conditionals is that we generally aim to avoid interpreting conditionals so that they are epistemically equivalent to their consequents, thus motivating the constraint that conditionals will only be interpreted relative to partitions on which they are non-trivial in the following sense:

$\ulcorner$ if $\mathrm{A}, \mathrm{B}\urcorner$ is trivial in $c$ relative to $\mathbf{Z}$ iff for any $w \in E_{c}: A \rightarrow_{c}^{\mathbf{Z}} B$ is true at $w$ iff $B$ is true at $w$.

(Where ' $A \rightarrow_{c}^{\mathbf{Z}} B$ ' denotes the proposition $\ulcorner$ if $\mathrm{A}, \mathrm{B}\urcorner$ would express in a context exactly like $c$ except possibly for it being interpreted relative to $\mathbf{Z}$ )

A crucial upshot of the non-triviality constraint is that we expect $\ulcorner$ if $A, B\urcorner$ to not be interpreted relative to the partition $\{B, \bar{B}\}$, since on this interpretation, it would be epistemically equivalent to its consequent. ${ }^{36}$

Before turning to the application of my theory to the cases described at the outset, I pause to note the following important consequence of Admissibility: whenever some non-trivial partition meets both constraints, the trivial partition $\left\{E_{c}\right\}$ will, but not vice versa. Given the contrapositive of Theorem 3-that Ramsey-violating conditionals must be interpreted relative to some partition other than the trivial one - already one may be

elucidates some of the places that are off limits (the barn, the shed, the highway), and also some of the possible hiding spots. With a pause and a look directly into Joe's eyes, he utters (14). In this context, I think it is very clear that the organizer has just told Joe that the treasure is in the attic. However, notice that this isn't a challenge to Avoid trivialization. The reason is that the motivation for the interpretive generalization is that normally we would just utter "The treasure is in the attic" if that's all we wanted to convey (since in this case it would be less liable to misinterpretation). But in this context, the organizer is not liable to misinterpretation (because of their previous deal) and furthermore has every reason not to utter "The treasure is in the attic" since doing so would ruin his plans. I think analogous cases can be constructed for conditionals that may violate my non-triviality constraint below, but I won't take more time on this point.

${ }^{36}$ Suppose $\ulcorner$ if $\mathrm{A}, \mathrm{B}\urcorner$ is interpreted relative to $\{B, \bar{B}\}$. Consider the class of worlds in $E_{c}$ as falling into four groups: $A B-, A \bar{B}-, \bar{A} B-$, and $(\bar{A} \wedge \bar{B})$-worlds. I'll show that for each $w \in E_{c}$ in each group, $\ulcorner$ if $\mathrm{A}, \mathrm{B}\urcorner$ is true at $w$ iff $B$ is true at $w$. Restrict attention to worlds in $E_{c}$. By Centering, $\ulcorner$ if $\mathrm{A}, \mathrm{B}\urcorner$ is true at every $A B$-world and false at every $A \bar{B}$-world. By Serious partitioning, $\ulcorner$ if $\mathrm{A}, \mathrm{B}\urcorner$ is true at every $\bar{A} B$-world (since the $c$-closest $A$-world to every such world will be an $A B$-world). Furthermore, $\ulcorner$ if $\mathrm{A}, \mathrm{B}\urcorner$ is false at every $(\bar{A} \wedge \bar{B}$ )-world (since the $c$-closest $A$-world to every such world will be an $A \bar{B}$-world). 
able to anticipate how my theory will predict the paucity of Ramsey-violating conditionals. I'll return to this point in $§ 5.3 .3$. In the next section, I turn to showing how combining my pragmatic theory with The SP theory predicts our observations about Ramsey-violating and Ramsey-friendly conditionals.

\subsection{Application}

\subsubsection{Ramsey-violating interpretations}

I turn now to show how my theory accounts for Ramsey-violating interpretations of the target conditionals in the contexts Hospital and Oracle.

\section{Hospital}

Begin with the following summary of the relevant probabilities given in Hospital and our target conditional (3):

$$
\begin{aligned}
& P(Z)=1 / 4 \quad Z=\text { that Jones is from Zephyr, } \bar{Z}=\text { that Jones is from Appleton } \\
& P(S)=1 / 3 \quad S=\text { that Jones is showing symptoms of B-flu } \\
& P(Z \mid S)=5 / 8 \quad B=\text { that Jones has B-flu } \\
& P(B \mid S Z)=9 / 10 \\
& P(B \mid S)=3 / 5
\end{aligned}
$$

(3) If Jones is showing symptoms of B-flu, he has B-flu.

$$
S \rightarrow_{c} B
$$

We calculate the probability that (3) is true in Hospital as follows:

$$
\begin{aligned}
& P\left(S \rightarrow_{c} B\right)=P\left(S \rightarrow_{c} B \mid S\right) \cdot P(S)+P\left(S \rightarrow_{c} B \mid \bar{S}\right) \cdot P(\bar{S}) \\
& =P(B \mid S) \cdot P(S)+P\left(S \rightarrow_{c} B \mid \bar{S}\right) \cdot P(\bar{S}) \\
& =P(B \mid S) \cdot P(S)+\left(\sum_{Z \in \mathbf{Z}_{c}} P(B \mid Z S) \cdot P(Z \mid \bar{S})\right) \cdot P(\bar{S})
\end{aligned}
$$

\section{From Centering}

From Prob

Now, what partition is (3) interpreted relative to in the context Hospital? By Default, we expect it to be the most salient admissible partition in the context. Which partitions are salient? $\left\{E_{c}\right\}$ is trivially, but so are the following questions which are under discussion given the information as stated: whether Jones is showing symptoms of B-flu $(\mathbf{S})$, whether Jones has B-flu (B), whether Jones is from Appleton or Zephyr $(\mathbf{Z})$. However, we know 
immediately that neither $\mathbf{S}$ nor $\mathbf{B}$ are admissible (the former violates the well-definedness criterion and the latter the non-triviality criterion). $\mathbf{Z}$, however, is admissible, since $\ulcorner$ if $\mathrm{S}, \mathrm{B}\urcorner$ is well-defined and non-trivial relative to $\mathbf{Z}$. Therefore, since $\mathbf{Z}$ is more salient than $\left\{E_{c}\right\}$ we predict by Default that the default interpretation of $(3)$ is relative to $\mathbf{Z}$. Thus, we have: ${ }^{37}$

$$
\begin{aligned}
& \sum_{Z \in \mathbf{Z}_{c}} P(B \mid Z S) \cdot P(Z \mid \bar{S})=P(B \mid Z S) \cdot P(Z \mid \bar{S})+P(B \mid \bar{Z} S) \cdot P(\bar{Z} \mid \bar{S}) \\
& =\quad 9 / 10 \cdot 1 / 16+1 / 10 \cdot 15 / 16 \\
& =9 / 160+15 / 160 \\
& =\quad 3 / 20
\end{aligned}
$$

Plugging this back into our calculation of $P\left(S \rightarrow_{c} B\right)$ above yields:

$$
\begin{aligned}
& P\left(S \rightarrow_{c} B\right)=P(B \mid S) \cdot P(S)+\left(\sum_{Z \in \mathbf{Z}_{c}} P(B \mid Z S) \cdot P(Z \mid \bar{S})\right) \cdot P(\bar{S}) \\
& =3 / 5 \cdot 1 / 3+3 / 20 \cdot 2 / 3 \\
& =\quad 3 / 15+6 / 60 \\
& =\quad 3 / 10
\end{aligned}
$$

Thus, we predict that the probability of (3) in Hospital is $3 / 10$, which is intuitively low, predicting our intuition about the example. Furthermore, this value is not equal to the conditional probability that Jones has B-flu, given that he shows symptoms of B-flu, which is $3 / 5$. Therefore, we predict that (3) is an exception to RAMSEY'S OBSERVATION, as desired. Thus, Default makes the correct prediction in this case.

\section{Oracle}

I restate the case and the target conditionals here:

Oracle. A highly reliable oracle is about to tell us whether a recently-tossed coin landed heads or tails. Since you know the coin to be weighted towards tails, you are pretty sure it landed tails. The oracle then makes her prediction, although you can't quite make out what she says. You are pretty sure she says "heads".

(4) a. If the oracle is correct, the coin landed heads.

$\ulcorner$ if $\mathrm{O}, \mathrm{H}\urcorner$

b. If the oracle is correct, then she said "heads".

$\left\ulcorner\right.$ if $\left.\mathrm{O}, \mathrm{S}_{\mathrm{H}}\right\urcorner$

${ }^{37} P(Z \mid \bar{S})=\frac{P(\bar{S} \mid Z) \cdot P(Z)}{P(\bar{S})}=\frac{1 / 6 \cdot 1 / 4}{2 / 3}=1 / 16$ 
Let $H$ denote that the coin landed heads (and $\bar{H}$ that it landed tails - thus, assume these are the only alternatives), $O$ that the oracle is correct, and $S_{H}$ that the oracle said "heads". Given the information in the case, you think that $O, \bar{H}$, and $S_{H}$ are each likely. Furthermore, given the setup, $P(H \mid O)=P\left(S_{H} \mid O\right)$, since $H$ and $S_{H}$ are equivalent at the $O$-region of epistemic space.

What does Default predict about the partitions we (by default) interpret (4-a) and (4-b) relative to in this context? First, notice that most plausibly the following questions are under discussion in Oracle: whether the oracle is correct $\mathbf{O}$, whether the coin landed heads $\mathbf{H}$, and whether the oracle said heads $\mathbf{S}_{\mathbf{H}}$. Next, notice that:

- For (4-a), $\ulcorner$ if $\mathrm{O}, \mathrm{H}\urcorner$ :

Both $\mathbf{O}$ and $\mathbf{H}$ are not admissible.

But $\mathbf{S}_{\mathbf{H}}$ is admissible (since $\ulcorner$ if $\mathrm{O}, \mathrm{H}\urcorner$ is well-defined and non-trivial relative to $\left.\mathbf{S}_{\mathbf{H}}\right)$.

- For (4-b), $\left\ulcorner\right.$ if $\left.\mathrm{O}, \mathrm{S}_{\mathrm{H}}\right\urcorner$ :

Both $\mathbf{O}$ and $\mathbf{S}_{\mathbf{H}}$ are not admissible.

But $\mathbf{H}$ is admissible (since $\left\ulcorner\right.$ if $\left.\mathrm{O}, \mathrm{S}_{\mathrm{H}}\right\urcorner$ is well-defined and non-trivial relative to H).

Thus, Default predicts that (4-a) will be interpreted relative to $\mathbf{S}_{\mathbf{H}}$ and (4-b) relative to H. We can therefore calculate their probabilities as follows:

$$
\begin{array}{ll}
P\left(O \rightarrow_{c} H\right)=P\left(O \rightarrow_{c} H \mid O\right) \cdot P(O)+P\left(O \rightarrow_{c} H \mid \bar{O}\right) \cdot P(\bar{O}) & \\
= & P(H \mid O) \cdot P(O)+P\left(O \rightarrow_{c} H \mid \bar{O}\right) \cdot P(\bar{O}) \\
= & P(H \mid O) \cdot P(O)+\left[P\left(O \mid S_{H} H\right) \cdot P\left(S_{H} \mid \bar{O}\right)+c\left(O \mid \overline{S_{H}} H\right) \cdot P\left(\overline{S_{H}} \mid \bar{O}\right)\right] \cdot P(\bar{O})
\end{array}
$$

From Prob

But now notice that:

$$
\begin{aligned}
& P\left(O \mid S_{H} H\right) \cdot P\left(S_{H} \mid \bar{O}\right)+P\left(O \mid \overline{S_{H}} H\right) \cdot P\left(\overline{S_{H}} \mid \bar{O}\right) \\
& =1 \cdot P\left(S_{H} \mid \bar{O}\right)+0 \\
& =P\left(S_{H} \mid \bar{O}\right)
\end{aligned}
$$


$P\left(O \mid S_{H} H\right)=1$ since you ought to be certain that the oracle is correct upon learning that the oracle said "heads" and that the coin landed heads. Therefore, substituting back into (*) yields:

$$
\begin{aligned}
& P\left(O \rightarrow_{c} H\right) \\
& =P(H \mid O) \cdot P(O)+P\left(S_{H} \mid \bar{O}\right) \cdot P(\bar{O}) \\
& =P\left(S_{H} \mid O\right) \cdot P(O)+P\left(S_{H} \mid \bar{O}\right) \cdot P(\bar{O}) \\
& =P\left(S_{H}\right)
\end{aligned}
$$

Since $P\left(S_{H}\right)$ is high, we predict that $P\left(O \rightarrow{ }_{c} H\right)$ is high too. Next,

$$
\begin{aligned}
& P\left(O \rightarrow_{c} S_{H}\right)=P\left(O \rightarrow_{c} S_{H} \mid O\right) \cdot P(O)+P\left(O \rightarrow_{c} S_{H} \mid \bar{O}\right) \cdot P(\bar{O}) \\
& =P\left(S_{H} \mid O\right) \cdot P(O)+P\left(O \rightarrow_{c} S_{H} \mid \bar{O}\right) \cdot P(\bar{O}) \quad \text { From Centering } \\
& =P\left(S_{H} \mid O\right) \cdot P(O)+\left[P\left(O \mid H S_{H}\right) \cdot P(H \mid \bar{O})+c\left(O \mid \bar{H} S_{H}\right) \cdot P(\bar{H} \mid \bar{O})\right] \cdot P(\bar{O})
\end{aligned}
$$

From Prob

But now notice that:

$$
\begin{aligned}
& P\left(O \mid H S_{H}\right) \cdot P(H \mid \bar{O})+P\left(O \mid \bar{H} S_{H}\right) \cdot P(\bar{H} \mid \bar{O}) \\
& =1 \cdot P(H \mid \bar{O})+0 \\
& =P(H \mid \bar{O})
\end{aligned}
$$

Therefore, substituting back into $(* *)$ yields:

$$
\begin{aligned}
& P\left(O \rightarrow_{c} S_{H}\right) \\
& =P\left(S_{H} \mid O\right) \cdot P(O)+P(H \mid \bar{O}) \cdot P(\bar{O}) \\
& =P(H \mid O) \cdot P(O)+P(H \mid \bar{O}) \cdot P(\bar{O}) \\
& =P(H)
\end{aligned}
$$

But since $P(H)$ is low, we have shown that my semantics predicts that $P\left(O \rightarrow_{c} S_{H}\right)$ is low. Thus, my theory predicts that $P\left(O \rightarrow_{c} H\right) \neq P\left(O \rightarrow_{c} S_{H}\right)$. Yet notice that $P(H \mid O)=P\left(S_{H} \mid O\right)$ since (as we already saw) $H$ and $S_{H}$ are epistemically equivalent given $O$. Therefore, my theory again correctly predicts the Ramsey-violating interpretations of $(4-a)$ and $(4-b)$ in Oracle. 


\subsubsection{Overriding Ramsey-violating interpretations}

We turn now to question (c): why, even evaluated against background information that would normally bias a Ramsey-violating interpretation for a conditional, can it sometimes receive a Ramsey-friendly one? As before, I'll focus on Kaufmann's example. Thus, imagine someone saying (in the context of Hospital):

"If Jones is showing symptoms of B-flu, then he is from Zephyr.

Therefore,

(3) If Jones is showing symptoms of B-flu, he has B-flu."

The reasoning is compelling. Thus, even though (3) by default seems to receive a Ramseyviolating interpretation in Hospital on which it is unlikely, once embedded in this line of reasoning, it no longer seems unlikely (on the contrary, it seems to follow that it is likely, given that (5) and (6) are both jointly likely).

Kaufmann took this argument to show that conditionals have both "local" and "global" interpretations, perhaps bolstering an ambiguity theory. However, as we saw in $\S 2$, Kaufmann's theory did not predict why we get the Ramsey-violating interpretation of (3) in Hospital and the Ramsey-friendly interpretation of (3) in the context of running the argument (5) and (6). Here's how my theory can predict this latter observation. I first assume that there is general pragmatic pressure to interpret the sentences uttered by individuals charitably - that is, to interpret them as faithful to their manifest communicative intentions as best you can, consistent with other constraints on interpretation. Then, whenever a speaker tokens an argument and indicates that she thinks the joint truth (or high probability of truth) of the premises is a reason to accept the truth (or high probability of truth) of the conclusion, charity puts pressure on us (as far as we can, consistent with other constraints on interpretation) to interpret her premises so that their joint truth (or high probability of truth) is in fact a reason to accept the truth (or high probability of truth) of her conclusion. In the case of the argument being run from (5) and (6) to (3), this involves interpreting the three conditionals relative to the same partition-otherwise, (5) and (6) both being likely would not be a reason to think that (3) is likely (since the argument would be equivocal). 
Next, notice that, of the questions plausibly under discussion in the context- $\mathbf{S}, \mathbf{Z}$, and $\mathbf{B}$ - both $\mathbf{S}$ and $\mathbf{Z}$ are inadmissible partitions for the first premise, (5) ( $\mathbf{S}$ is ruled out by well-definedness, and $\mathbf{Z}$ by non-triviality). This leaves two possible partitions to interpret (5) relative to: $\mathbf{B}$ and the trivial partition $\left\{E_{c}\right\}$. However, notice that the second premise, (6), is trivial relative to $\mathbf{B}$. Therefore, the only way to interpret (5), (6), and (3) relative to the same partition without trivializing any of them is to interpret each relative to $\left\{E_{c}\right\} .{ }^{38}$ Since we aim to interpret speakers charitably (consistent with other constraints on interpretation), the theory predicts that we will interpret (5), (6), and (3) relative to $\left\{E_{c}\right\}$. But then, given Theorem 2, we know that any conditional interpreted relative to $\left\{E_{c}\right\}$ will be Ramsey-friendly. Thus, my theory predicts, correctly, that in the context of this speaker's argument, (3) is Ramsey-friendly and thus likely true. ${ }^{39,40}$

\footnotetext{
${ }^{38}$ We might wonder whether we can hear a reading of (5) in which it is interpreted relative to $\mathbf{B}$ at all. I hypothesize that we do get this reading, but that we mistakenly think that $\mathbf{B}$ does not probabilistically depend on $S$ because of how the information is presented in Hospital. As such, we think that on this reading $\ulcorner$ if $\mathrm{S}, \mathrm{Z}\urcorner$ is Ramsey-friendly (and hence likely). I'll discuss Theorem 4, which ensures this result of my theory, in $§ 5.3 .3$. For now, I want to offer some evidence for my hypothesis. If we present the same information as Hospital by distributing the frequencies across $\mathbf{B}$ instead of $\mathbf{Z}$, I submit that we do find that $\ulcorner$ if $\mathrm{S}, \mathrm{Z}\urcorner$ is Ramsey-violating on this interpretation:

Patients with B-flu Patients without B-flu

10 total 62 total

All symptomatic 10 symptomatic

$\circ 1$ from Appleton $\circ 9$ from Appleton

$\circ 9$ from Zephyr $\quad 1$ from Zephyr

Presented in this way, (5) seems intuitively unlikely. Why? Well, Jones most likely doesn't have B-flu. But in that case if he's showing symptoms of B-flu, he's probably from Appleton, not Zephyr. So, most likely (5) is false. This is some evidence that my hypothesis above is correct-we can hear $\ulcorner$ if $\mathrm{S}, \mathrm{Z}\urcorner$ as interpreted relative to $\mathbf{B}$ but we simply think it is Ramsey-friendly on that interpretation. Of course, there is an alternative hypothesis, which is that $\ulcorner$ if $\mathrm{S}, \mathrm{Z}\urcorner$ cannot be interpreted relative to $\mathbf{B}$ in Hospital, but it can be interpreted relative to $\mathbf{B}$ when the frequencies of the case are presented in this way. I won't try to argue against this counter-hypothesis at this time.

${ }^{39}$ What about the Ramsey-friendly reading of the oracle-sentences (4-a) and (4-b) when embedded in the following line of reasoning:

(i) a. If the oracle is correct, the coin landed heads.

b. If the oracle is correct, then the coin landed heads if and only if she said 'heads'.

c. So, if the oracle is correct, she said 'heads'.

Someone rehearsing this argument clearly intends that each conditional be interpreted relative to the same partition. However, the only admissible partition for both (4-a) and (4-b) is the trivial one $\left\{E_{c}\right\}$. Thus, we may offer the same explanation as above for the intuition that (4-a)/(4-b) are equally likely in the context of this argument, even though we do not know how likely either is.

${ }^{40}$ An anonymous reviewer wonders whether my explanation here will incorrectly predict that (3) will receive a Ramsey-friendly interpretation when uttered (in the Hospital scenario) as follows:
} 
I want to emphasize that this is just one way that Default may be overridden. I am open to there by other ways to do so, however evaluating every possible way goes far beyond the scope of this paper. My aim here is just to articulate a theory that makes predictions about several cases, and offer a compelling explanation of what is going on in those cases. In that regard, I think my theory has been quite successful. I am hopeful that further refinements of the theory will continue to yield adequate predictions when we expand our attention to other kinds of cases.

\subsubsection{The rarity of Ramsey-violating interpretations}

My theory predicts the rarity of Ramsey-violating interpretations of conditionals because it predicts that contexts in which some non-trivial partition is salient and admissible for a conditional $\ulcorner$ if $A, B\urcorner$ are rare. Given Theorem 3 (which states that conditionals interpreted relative to the trivial partition will be Ramsey-friendly) and our claims about salient partitions in $\S 5.1$, this means that my theory predicts that a conditional will receive a Ramsey-violating interpretation only if there is a question under discussion in the context which is an admissible partition for that conditional. We have every reason to expect that such contexts will be rare. However, I want to draw attention to one more theorem, which shows that it is even harder for conditionals to receive Ramsey-violating interpretations on my theory:

Theorem 4. $\ulcorner$ if $\mathrm{A}, \mathrm{B}\urcorner$ is Ramsey-violating in $c$ iff for some $Z \in \mathbf{Z}_{c}: P(Z \mid A) \neq$ $P(Z \mid \bar{A})$ (where $\mathbf{Z}_{c}$ is the background partition for $\ulcorner$ if $\mathrm{A}, \mathrm{B}\urcorner$ in $c$ ).

Theorem 4 adds another hurdle that Ramsey-violating conditionals must clear-they must be such that the admissible QUD they are interpreted relative to is such that it probabilistically depends on the conditional's antecedent.

(i) If Jones is from Zephyr, then he's from Zephyr.

(3) So, if Jones is showing symptoms of B-flu, he has B-flu.

Just as with (5), (6), (3), here, the speaker here seems to indicate that she thinks that (i) being true (or likely) is a reason to think that (3) is true (or likely). However, my intuition is that (3) still has its default, Ramsey-violating, interpretation (and is thus unlikely). Notice that the theory sketched above does not predict otherwise. It entails that interpretive charity puts pressure on us (as far as we can, consistent with other constraints on interpretation) to interpret (i) and (3) so that the likelihood (or truth) of the former is a reason to think that the latter is likely (or true). But there is no way of interpreting these two sentences in which that is the case. Therefore, since no other interpretive considerations are salient, the theory predicts (3) should have its default, Ramsey-violating, interpretation, which seems correct. 
We can verify that this condition is met in our two examples. For instance, recall the conditional (3) from Hospital:

If Jones is showing symptoms of B-flu, he has B-flu.

$S \rightarrow B$

In Hospital, the question whether Jones is from Zephyr, $\mathbf{Z}$, is under discussion and admissible for (3). Furthermore, the likelihood that Jones is from Zephyr depends on whether he is showing symptoms of B-flu. Recall the two conditionals (4-a) and (4-b) from Oracle:

a. If the oracle is correct, the coin landed heads.

$$
O \rightarrow H
$$

b. If the oracle is correct, the oracle said "heads".

$$
O \rightarrow S_{H}
$$

In Oracle, the question of whether the oracle said "heads", $\mathbf{S}_{\mathbf{H}}$, is under discussion and admissible for (4-a). Furthermore, the likelihood that the oracle said "heads" depends on whether the oracle is correct. Similarly, in that context the question of whether the coin landed heads, $\mathbf{H}$, is also under discussion and admissible for (4-b). Furthermore, the likelihood that the coin landed heads depends on whether the oracle is correct.

Thus, given the constraints on non-default background partitions built into Override, along with Theorem 3, my theory predicts that exceptions to RAMSEY'S OBSERVATION arise only in these special contexts. Furthermore, it is reasonable to expect that these contexts will be very rare, since they require the introduction of a question under discussion that meets very severe restrictions. Therefore, my theory predicts that the exceptions to RAMSEY'S OBSERVATION ought to be rare. ${ }^{41}$

\section{Predicting Prob}

We have now seen that combining The SP theory with the pragmatics Admissible and Default yields a theory flexible enough to predict our intuitions about Ramsey-friendly and Ramsey-violating conditionals in a range of cases, thus providing plausible answers to our questions (a)-(d). However, throughout the discussion of $\S 5$, we appealed to the

\footnotetext{
${ }^{41}$ One might wonder then how my theory, which is avowedly propositionalist, can predict few exceptions to RAMSEY'S OBSERVATION in light of Lewis's triviality proofs ([lewis:1976, lewis:1986, stalnaker:1976, gibbard:1981, hajek:1989, hajek:1994, hajek:2011b, hajek:2012, hajekhall:1994, edgington:1995, bennett:2003]) that the probability of a conditional can only equal its corresponding conditional probability in full generality in the trivial case (in which the probability of the conditional is equal to the probability of its consequent). I refer the interested reader to [khoo:2013c], which attempts to diffuse the problem raised by these triviality proofs for propositionalist theories of indicative conditionals that predict instances of RAMSEY'S OBSERVATION.
} 
principle Prob, which I only gave a minimal motivation for in $\S 4$. In this final section, I aim to make good on my promise about predicting Prob by merging The SP theory with a concrete proposal about the indeterminacy exhibited by conditionals at worlds where their antecedents are false. In fact, I'll make good on this twice over by showing two different ways this can be done.

\subsection{Indeterminacy as ignorance}

Recall that The SP theory is the conjunction of Stalnaker semantics, Centering, and Serious partitioning:

\section{Stalnaker semantics:}

$\ulcorner$ if $\mathrm{A}, \mathrm{B}\urcorner$ is true at $c, w$ iff $\mathrm{B}$ is true at $c, w^{\prime}$; where $w^{\prime}$ is the $c$-closest epistemically accessible $_{c} A$-world to $w$

Centering: if $A$ is true at $w$, then the $c$-closest $A$-world to $w$ is $w$.

Serious partitioning: where $\mathbf{Z}_{c}$ is the partition $c$ assigns to $\ulcorner$ if $A, B\urcorner$ : the $c$-closest $A$-world to $w$ is among $[w] \mathbf{z}_{c}$

Let $\mathcal{F}_{c}^{A}$ be the set of admissible $A$-closeness functions determined by $c$, constrained in accordance with Centering and Serious partitioning. These constraints allow for contexts $c$ in which there is a world $w \in E_{c}$ such that there are two $A$-closeness functions $f_{1}^{A}$ and $f_{2}^{A}$ in $\mathcal{F}_{c}^{A}$ such that $f_{1}^{A}(w)$ is a $B$-world and $f_{2}^{A}(w)$ is a $\bar{B}$-world. In such a context, The SP theory predicts that $\ulcorner$ if $\mathrm{A}, \mathrm{B}\urcorner$ is indeterminate at $w$. The first implementation I sketch here takes this indeterminacy to be epistemic in nature, arising from our in principle ignorance about the workings of our language. ${ }^{42}$ The proposal is that knowing what conditionals mean involves (tacitly) knowing the following:

(E1) $\mathcal{F}_{c}^{A}$ is the maximal set of $A$-closeness functions which contains only functions (i) defined only on worlds in $E_{c}$, (ii) that map each $A$-world in $E_{c}$ to itself, and (iii) which, for each $Z \in \mathbf{Z}$, map any $\bar{A} Z$-world to a $Z$-world.

\footnotetext{
${ }^{42}$ This view has much in common with so-called epistemicist views in vagueness, in which vague predicates have fully determinate extensions, though we are ignorant, perhaps in principle, about whether various objects fall in their extensions (see esp. [sorensen:1988a, sorensen:2001, williamson:1994]). See also [mcdermott:1999, hawthorne:2005, bradley:2012] for similar approaches to conditional indeterminacy.
} 
(E2) Exactly one function in $\mathcal{F}_{c}^{A}$ is in fact operative in $c$ (that is, determines the proposition expressed by $\ulcorner$ if $\mathrm{A}, \mathrm{B}\urcorner$ in $c$ ).

(E3) Conditional on any evidence one could possibly learn, each function in $\mathcal{F}_{c}^{A}$ is as likely as any other in $\mathcal{F}_{c}^{A}$ to be operative in $c .^{43,44}$

In what follows, I will derive an epistemicist version of Prob from the assumption that (E1)-(E3) are tacitly known. ${ }^{45}$

To do so precisely, we need some new terminology. Let $\llbracket \rrbracket$ be an interpretation function, which maps expressions of the target language (English) to their extensions relative to a context and world. Thus, $\llbracket \rrbracket$ maps sentences to truth values (either 1 or 0 ) relative to a context and a world. What I previously called the proposition expressed by a sentence A in context $c$, we define as the horizontal proposition of $\mathrm{A}$ at $c$ as follows:

For any sentence $\mathrm{A}$ and context $c$, the horizontal proposition expressed by $\mathrm{A}$ at $c$ is $\llbracket \mathrm{A} \rrbracket^{c}={ }_{\operatorname{def}}\left\{w: \llbracket \mathrm{A} \rrbracket^{c, w}=1\right\}$.

Next, we define a counterpart context $c[w]$ to be a possible context which is like $c$ in various respects, except that it takes place at $w$ (instead of the world of $c$ ). We can then define the diagonal proposition of $\mathrm{A}$ at $c$ as follows:

\footnotetext{
${ }^{43}$ I won't go into the details as to why this might be. For one possible motivation (regarding subtle differences in the patterns of use of vague predicates), see [williamson:1994]. I also want to highlight that I am not here defending epistemicism, but rather showing how an epistemicist implementation of Stalnaker semantics can predict an analogous version of Prob. I want to officially remain neutral on the choice of indeterminacy theory in this paper.

${ }^{44}$ I'm here also assuming that the probability distribution is uniform. If our probability distribution is not uniform, then we would need to generalize (E3) so that functions mapping to worlds which are comparatively more likely are themselves comparatively more likely. Thus, where $f_{w}^{A}$ is the $A$-closeness function operative at $c[w]$, and $p$ is a probability mass function which maps worlds to the likelihood of their being actual (cf. [lewis:1981c, gardenfors:1988]), we hold that any worlds $w, w^{\prime}$ such that $f_{w}^{A}(w)=w^{\prime}$ are such that $p(w)=p\left(w^{\prime}\right)$. In what follows, I will assume that the probability distribution is uniform, and thus sidestep this complication. Thanks to an anonymous reviewer for pressing me on this point.

${ }^{45}$ It might seem that such a result cannot obtain, given [hajek:1989]'s triviality result. Hájek's proof establishes that for any $P$ defined over a finite set of possible worlds (with cardinality greater than 2), there will be more conditional probability values than unconditional probability values. However, if Prob holds, and every conditional in some context $c$ receives the trivial partition $E_{c}$, my theory predicts that every conditional will be Ramseyan-just what Hájek's proof demonstrates cannot be the case, if $E_{c}$ is finite. Without going into the details of the proof, my response to this problem is to accept the consequence that in contexts in which every conditional is assigned the trivial partition, the set of epistemically accessible worlds is infinite. That this is a possible move to make has already been established in the tenability proof of [fraassen:1976]. See also [mcgee:1989, kaufmann:2009a, khoo:2013c] for further discussion.
} 
For any sentence $\mathrm{A}$ and context $c$, the diagonal proposition expressed by $\mathrm{A}$ at $c$ is $\dagger^{c}[\mathrm{~A}]=_{\operatorname{def}}\left\{w \in E_{c}: \llbracket \mathrm{A} \rrbracket^{c[w], w}=1\right\}$.

$c[w]$ will be defined on each world in $E_{c}$, since at each such world there is a counterpart conversation of the actual conversation taking place. Thus, the diagonal of $\mathrm{A}$ is true at some world $w \in E_{c}$ iff the horizontal proposition $\mathrm{A}$ would have expressed had it been uttered at $c[w]$ would be true at $w . \llbracket \mathrm{A} \rrbracket^{c} \neq \dagger^{c}[\mathrm{~A}]$ iff $\mathrm{A}$ expresses different horizontal propositions at (the counterpart contexts of) different worlds in $E_{c}$ - that is, if and only if there is ignorance with respect to what horizontal proposition A expresses as uttered in c. We now characterize the horizontal/diagonal propositions expressed by $\ulcorner$ if $\mathrm{A}, \mathrm{B}\urcorner$ at $c$, given Stalnaker semantics and (E1):

$\llbracket\ulcorner$ if $\mathrm{A}, \mathrm{B}\urcorner \rrbracket^{c}=\left\{w \in E_{c}: w^{\prime} \in B\right.$; where $w^{\prime}$ is the $c$-closest $A$-world to $w$ in $\left.E_{c}\right\}$

$\dagger^{c}[\ulcorner$ if $\mathrm{A}, \mathrm{B}\urcorner]=\left\{w \in E_{c}: w^{\prime} \in B\right.$; where $w^{\prime}$ is the $c[w]$-closest $A$-world to $w$ in $\left.E_{c}\right\}$

Following [stalnaker:1978], we suppose that whenever there is uncertainty about which horizontal proposition is expressed by a sentence $\mathrm{A}$ in a context $c$, the asserted content of that sentence in that context is its corresponding diagonal proposition. Relatedly, we can calculate the probability that a sentence is true in a context as the expectation that it expresses a truth in that context as follows. Thus, where ' $\operatorname{Pr}_{c}(\mathrm{~A})$ ' ' is shorthand for 'the probability that $\mathrm{A}$ is true in $c^{\prime}$ :

DIAGONAL BRIDGE

a. $\operatorname{Pr}_{c}(\mathrm{~A})=P_{c}\left(\dagger^{c}[\mathrm{~A}]\right)$

b. $\operatorname{Pr}_{c}(\mathrm{~B} \mid \mathrm{A})=P_{c}\left(\dagger^{c}[\mathrm{~B}] \mid \dagger^{c}[\mathrm{~A}]\right)$

The version of Prob that I'll derive is:

Prob-E: $\operatorname{Pr}_{c}\left(\dagger^{c}[\ulcorner\right.$ if A, B $] \mid$ not-A $)=\sum_{Z \in \mathbf{Z}_{c}} P_{c}(B \mid Z A) \cdot P_{c}(Z \mid \bar{A})$

I assume throughout that there is no uncertainty about what proposition A expresses (so that $\left.\llbracket \mathrm{A} \rrbracket^{c}=\dagger^{c}[\mathrm{~A}]\right)$, and no uncertainty regarding the background partition for $\ulcorner$ if $\mathrm{A}, \mathrm{B}\urcorner$ in $c$ (so that $\forall w \in E_{c}: \mathbf{Z}_{c}=\mathbf{Z}_{c[w]}$ ). Thus, from DiAgOnAL BRIDGE and the law of total probability:

$$
\operatorname{Pr}_{c}(\ulcorner\text { if A, B }\urcorner \mid \text { not-A })=\sum_{Z \in \mathbf{Z}_{c}} P_{c}\left(\dagger^{c}[\ulcorner\text { if A, B }\urcorner] \mid Z \bar{A}\right) \cdot P_{c}(Z \mid \bar{A})
$$


Recall that $\dagger^{c}[\ulcorner$ if $\mathrm{A}, \mathrm{B}\urcorner]$ is true at $w$ iff the $c[w]$-closest $A$-world to $w$ is a $B$-world. As a helpful piece of terminology, for each $w \in E_{c}$, let $f_{w}^{A}$ be the $A$-closeness function that is operative at $c[w]$. Then, say that the $A$-world that some world $w \in E_{c}$ maps itself to is $f_{w}^{A}(w)$. Then, the probability of $\dagger^{c}[\ulcorner$ if $\mathrm{A}, \mathrm{B}\urcorner]$ given $Z \bar{A}$ just is the likelihood of being at a $Z \bar{A}$-world which maps itself to a $Z A B$-world. What we will show is that, given our assumptions above, the likelihood of being at a $Z \bar{A}$-world which maps itself to a $Z A B$-world just is the probability of $B$ given $Z A$.

Suppose that we learn $Z \bar{A}$. By (E1), we know that the admissible $A$-closeness functions will all map $Z \bar{A}$-worlds to $Z A$-worlds. And by (E2) and (E3), we know that each function in $\mathcal{F}_{c}^{A}$ is equally likely (with probability greater than 0 ) to be the closeness function operative in $c$. Hence, by (E1) and (E3), we know that each $Z A$-world $w$ will be such that some $Z \bar{A}$-world maps itself to $w$. Thus, we may partition $Z \bar{A}$ according to which world in $Z A$ the $Z \bar{A}$-worlds map themselves to. Let $\mathbf{X}$ be such a partition on $Z \bar{A}$. It follows that:

(i) $\forall X \in \mathbf{X}: \exists ! w \in A Z: \forall w^{\prime} \in X: f_{w^{\prime}}^{A}\left(w^{\prime}\right)=w$, and

(ii) $\forall w \in A Z: \exists ! X \in \mathbf{X}: \forall w^{\prime} \in X: f_{w^{\prime}}^{A}\left(w^{\prime}\right)=w$.

(i) states that for each cell of $\mathbf{X}$, there is exactly one $A Z$-world that every world in that cell maps itself to. (ii) states that for every world in $A Z$, there is exactly one cell of $\mathbf{X}$ such that every world in that cell maps itself to that $A Z$-world. Furthermore, it follows from (E3) that:

$$
\forall X, X^{\prime} \in \mathbf{X}: P_{c}(X \mid Z \bar{A})=P_{c}\left(X^{\prime} \mid Z \bar{A}\right)
$$

Next, let $\mathbf{X}_{B}$ be the set of cells in $\mathbf{X}$ comprised of worlds that all map themselves to a $Z A B$-world. Thus, since (by assumption) $P_{c}$ is uniform, follows from (i), (ii), and (20) that:

$$
\sum_{X \in \mathbf{X}_{B}} P_{c}(X \mid Z \bar{A})=P_{c}(B \mid Z A)
$$

Furthermore, we know that:

$$
\forall X \in \mathbf{X}: P_{c}\left(\dagger^{c}[\ulcorner\text { if } \mathrm{A}, \mathrm{B}\urcorner] \mid X\right)=
$$
a. 1 if $X \in \mathbf{X}_{B}$
(since then every world in $X$ maps itself to a $B$-world)
b. 0 if $X \notin \mathbf{X}_{B}$
(since then every world in $X$ maps itself to a $\bar{B}$-world) 
Finally, because $\mathbf{X}$ partitions $Z \bar{A}$, by the law of total probability:

$$
P_{c}\left(\dagger^{c}[\ulcorner\text { if } \mathrm{A}, \mathrm{B}\urcorner] \mid Z \bar{A}\right)=\sum_{X \in \mathbf{X}} P_{c}\left(\dagger^{c}[\ulcorner\text { if } \mathrm{A}, \mathrm{B}\urcorner] \mid X\right) \cdot P_{c}(X \mid Z \bar{A})
$$

And thus it follows from (21), (22), and (23) that:

$$
\sum_{X \in \mathbf{X}} P_{c}\left(\dagger^{c}[\ulcorner\text { if } \mathrm{A}, \mathrm{B}\urcorner] \mid Z \bar{A}\right)=P_{c}(B \mid Z A)
$$

And thus Prob-E follows from (19) and (24).

\subsection{Metaphysical indeterminacy}

I emphasize that epistemicism is not the only solution to predicting the right probabilities for conditionals, given Stalnaker semantics. In this section, I will briefly sketch an alternative implementation of my semantics, which yields an analogous version of Prob. This alternative implementation resembles the theories of [jeffrey:1991, stalnaker:1994, kaufmann:2004, kaufmann:2005b, kaufmann:2009a, schulz:2014a, ?]. On this implementation, for any $\bar{A} Z$-world $w$, we treat the indeterminacy in which $A Z$-world is the $c$-closest $A$-world to $w$ as metaphysical in nature. That is, we suppose that the world does not determine which of such worlds is the closest $A$-world to $w$; instead it determines a chance, for each $A Z$-world, that that world will be the $c$-closest $A$-world to $w$. Implementing this thought formally requires complicating the denotation of $\ulcorner$ if $\mathrm{A}, \mathrm{B}\urcorner$ at $c$, since whatever it denotes is no longer true or false relative to a possible world. One natural way to implement the idea is to let sentences denote random variables (that is, function from worlds to values in the unit interval $[0,1]$ rather than truth values) in contexts. Then, Stalnaker semantics and Serious partitioning would motivate the following denotation for $\ulcorner$ if A, $\mathrm{B}\urcorner$ (here $\llbracket \rrbracket$ assigns sentences values in unit interval relative to contexts and worlds): ${ }^{46}$

$$
\llbracket\ulcorner\text { if } \mathrm{A}, \mathrm{B}\urcorner \rrbracket^{c, w}=\left\{\begin{array}{lll}
\llbracket \mathrm{B} \rrbracket^{c, w} & \text { if } & \llbracket \mathrm{A} \rrbracket^{c, w}=1 \\
P_{c}(B \mid Z A) & \text { if } & \llbracket \mathrm{A} \rrbracket^{c, w}=0, w \in Z \\
& & \left(\text { Where } Z \in \mathbf{Z}_{c}\right)
\end{array}\right\}
$$

We then calculate the probability of a sentence in a context as the expectation of its likelihood (and do the same for conditional probabilities), as follows (let ' $\llbracket \mathrm{A} \rrbracket^{c}=x$ ' denote

\footnotetext{
${ }^{46}$ For an alternative yet formally equivalent implementation, see the discussion in [khoo:2013c], section 1.4 .
} 
$\left\{w: \llbracket \mathrm{A} \rrbracket^{c, w}=x\right\}$, and ' $R_{c}\left(\llbracket \mathrm{A} \rrbracket^{c}\right)^{\prime}$ ' denote the range of $\left.\llbracket \mathrm{A} \rrbracket^{c}\right)$ :

BRIDGE

$$
\begin{aligned}
& \text { a. } \operatorname{Pr}_{c}(\mathrm{~A})=\sum_{x \in R_{c}\left(\llbracket \mathrm{A} \rrbracket^{c}\right)} x \cdot P_{c}\left(\llbracket \mathrm{A} \rrbracket^{c}=x\right) \\
& \text { b. } \operatorname{Pr}_{c}(\mathrm{~B} \mid \mathrm{A})=\sum_{x \in R_{c}\left(\llbracket \mathrm{B} \rrbracket^{c}\right)} x \cdot P_{c}\left(\llbracket \mathrm{B} \rrbracket^{c}=x \mid \llbracket \mathrm{A} \rrbracket^{c}=1\right)
\end{aligned}
$$

Finally, we see that RANDOM and BRIDGE together entail the analogous version of Prob. From BRIDGE we have:

$$
\operatorname{Pr}_{c}(\ulcorner\text { if } \mathrm{A}, \mathrm{B}\urcorner \mid \text { not- } \mathrm{A})=\sum_{x \in R_{c}\left(\llbracket\ulcorner\text { if } \mathrm{A}, \mathrm{B}\urcorner \rrbracket^{c}\right)} x \cdot P_{c}\left(\llbracket\ulcorner\text { if } \mathrm{A}, \mathrm{B}\urcorner \rrbracket^{c}=x \mid \llbracket \text { not- } \mathrm{A} \rrbracket^{c}=1\right)
$$

Assuming $\mathrm{B}$ is not a conditional, values in the range of $\llbracket\ulcorner$ if $\mathrm{A}, \mathrm{B}\urcorner \rrbracket^{c}$ are 1,0 , and $P_{c}(B \mid Z A)$,

for each $Z \in \mathbf{Z}_{c}$. Since we care only about the values for $\llbracket\ulcorner$ if $\mathrm{A}, \mathrm{B}\urcorner \rrbracket^{c, w}$ at $\bar{A}$-worlds, the relevant range will include only the values $P_{c}(B \mid Z A)$, for each $Z \in \mathbf{Z}_{c}$. Furthermore, by RANDOM:

$$
\forall Z \in \mathbf{Z}_{c}:\left\{w \in \bar{A}: \llbracket\ulcorner\text { if } \mathrm{A}, \mathrm{B}\urcorner \rrbracket^{c, w}=P_{c}(B \mid Z A)\right\}=\{w \in \bar{A}: w \in Z\}
$$

Thus, we may substitute into (26) accordingly, yielding:

$$
\operatorname{Pr}_{c}(\ulcorner\text { if A, B }\urcorner \mid \text { not-A })=\sum_{Z \in \mathbf{Z}_{c}} P(B \mid Z A) \cdot P(Z \mid \bar{A})
$$

And this is the analogous version of Prob. Therefore, we can understand and appreciate the Kaufmann and Stalnaker \& Jeffery proposals to treat conditionals as random variables as implementations of Stalnaker semantics in ways that resolve the predicted indeterminacies as giving rise to objective chances rather than truth values. For the purposes of this paper, I want to remain neutral on the choice of indeterminacy framework within which we implement my semantic theory.

\section{Conclusion}

We began with some puzzling data about Ramsey-friendly and Ramsey-violating interpretations of conditionals (§1). I argued that, though a crucial step in the right direction, Kaufmann's partition-dependent theory of the probabilities of conditionals faces several challenges (§2). After thinking about the relationship between the truth conditions of 
conditionals and their probabilities at an abstract level (§3), I discussed a motivation for a well-known theory of conditionals due to Stalnaker ( $\$ 4)$. In the rest of that section, I motivated a generalization of that theory which resulted in a theory on which the truth conditions (and hence probabilities) of conditionals are partition-relative. This led me to think about the pragmatics of partitions assigned to conditionals in $\S 5$, and there I provided independent motivation for a theory which I argued made the right predictions about the puzzling data from $\S 1$. Finally, in $\S 6$, I showed how integrating my theory with a suitable theory of indeterminacy allows us to derive a suitable version of Prob, which is the crucial lemma the theory needs to predict the right probabilities of conditionals.

I argued that my semantic/pragmatic theory offers compelling answers to our questions (a)-(d) while avoiding the other challenges facing Kaufmann's theory discussed in $§ 1$.

a. What is the nature of the Ramsey-friendly and Ramsey-violating interpretations of conditionals? (Is it an ambiguity, for instance?)

b. Why do certain conditionals favor Ramsey-violating interpretations in certain contexts?

c. Why, in such contexts, can we sometimes override the Ramsey-violating interpretations in favor of Ramsey-friendly interpretations?

d. Why are Ramsey-violating interpretations of conditionals so rare?

My theory's answer to (a) is that the truth conditions of conditionals are determined relative to a salient partition which may vary from context to context. My pragmatic theory predicts that the partition that Ramsey-violating conditionals are interpreted relative to is a salient question under discussion meeting certain independently motivated constraints, rather than an objective-chance determining variable that the conditional's antecedent is causally independent of (as Kaufmann predicts). As such, my theory does not predict that conditionals can only be interpreted relative to such partitions, nor does it predict that the truth value of every indicative conditional at worlds where its antecedent is false goes by the truth value of its subjunctive counterpart at those worlds - both positive features, as I argued in $\S 2$. My theory's answer to (b) was the fact that conditionals are by default interpreted relative to salient questions under discussion (which meet the constraints given in Admissibility). The theory answers (c) by allowing that this default may be overridden by considerations of charity in speaker interpretation, as when a speaker clearly intends 
the sentences she utters to be such that the truth (or likelihood) of some of them is a reason for thinking that another of them is true (or likely). Finally, my theory answers (d) by entailing that the conditions under which a conditional will receive a Ramsey-violating interpretation are quite severe.

Thus, I hope to have motivated my theory both on independent grounds, the fact that it entails plausible answers to our questions (a)-(d), and the fact that its predictions are empirically correct. Of course, more work still remains to refine the theory and extend it to cases not yet explored. But I hope that the proposal here constitutes a step in the right direction in our thinking about the rare but genuine (and deeply puzzling) counterexamples to RAMSEY'S OBSERVATION. ${ }^{47}$

\section{Appendix}

\section{Proofs of Theorems}

The proofs of Theorem 1-Theorem 4 all make use of the following equation, which follows from the law of total probability:

$$
\begin{aligned}
& P\left(A \rightarrow_{c} B\right) \\
& =P\left(A \rightarrow_{c} B \mid A\right) \cdot P(A)+P\left(A \rightarrow_{c} B \mid \bar{A}\right) \cdot P(\bar{A}) \\
& =P(B \mid A) \cdot P(A)+P\left(A \rightarrow_{c} B \mid \bar{A}\right) \cdot P(\bar{A})
\end{aligned}
$$

Theorem 1. $\ulcorner$ if $\mathrm{A}, \mathrm{B}\urcorner$ is Ramsey-friendly in $c$ iff $P\left(A \rightarrow_{c} B \mid \bar{A}\right)=P(B \mid A)$.

Left-to-right. Suppose $\ulcorner$ if $\mathrm{A}, \mathrm{B}\urcorner$ is Ramsey-friendly in $c$, hence that $P\left(A \rightarrow_{c} B\right)=P(B \mid A)$. Then from (EQ) it follows that $P(B \mid A)=P(B \mid A) \cdot P(A)+P\left(A \rightarrow_{c} B \mid \bar{A}\right) \cdot P(\bar{A})$. Now we prove that $x=x y+z(1-y)$ only if $x=z$ :

Proof.

$$
x=x y+z(1-y)
$$

\footnotetext{
${ }^{47}$ I'd like to thank the following people for invaluable feedback on this paper (often at multiple stages of its development): Phil Bricker, Dan Greco, Larry Horn, Stefan Kaufmann, Laura Khoo, Mark Maxwell, Vann McGee, Chris Meacham, Aaron Norby, Bernhard Salow, Bob Stalnaker, Zoltán Szabó, Steve Yablo, Ian Wells, and Bruno Whittle. I am also grateful for the feedback of three anonymous reviewers for Linguistics ES Philosophy, which helped improve the paper in many dimensions.
} 


$$
\begin{aligned}
& x-x y=z(1-y) \\
& x(1-y)=z(1-y) \\
& x=z
\end{aligned}
$$

Therefore, $P\left(A \rightarrow_{c} B \mid \bar{A}\right)=P(B \mid A)$.

Right-to-left. Suppose $P\left(A \rightarrow_{c} B \mid \bar{A}\right)=P(B \mid A)$. Substituting into (EQ) yields: $P\left(A \rightarrow_{c}\right.$ $B)=P(B \mid A) \cdot P(A)+P(B \mid A) \cdot P(\bar{A})$. We prove below that $x y+x(1-y)=x$ :

Proof.

$$
\begin{aligned}
& x y+x(1-y)= \\
& x y+x-x y= \\
& x
\end{aligned}
$$

Therefore, $P\left(A \rightarrow_{c} B\right)=P(B \mid A)$.

Theorem 2. $P\left(A \rightarrow_{c} B\right)=P\left(A \rightarrow_{\ell}^{\mathbf{Z}_{c}} B\right)$; where $\mathbf{Z}_{c}$ is the partition assigned to $A \rightarrow_{c} B$.

Proof. I assume that The SP theory governs $A \rightarrow_{c} B$. Begin with the following equations:

$$
\begin{array}{ll}
\text { a. } & P\left(A \rightarrow_{c} B \mid A\right)=P(B \mid A) \\
\text { b. } & P\left(A \rightarrow_{c} B \mid \bar{A}\right)=\sum_{Z \in \mathbf{Z}_{c}} P(B \mid Z A) \cdot P(Z \mid \bar{A}) \\
\text { c. } & P\left(A \rightarrow_{\ell}^{\mathbf{Z}_{c}} B\right)=\sum_{Z \in \mathbf{Z}_{c}} P(B \mid Z A) \cdot P(Z)
\end{array}
$$

From (EQ), Centering and Prob we have:

$$
\begin{aligned}
P\left(A \rightarrow_{c} B\right) & =P\left(A \rightarrow_{c} B \mid A\right) \cdot P(A)+P\left(A \rightarrow_{c} B \mid \bar{A}\right) \cdot P(\bar{A}) \\
& =P(B \mid A) \cdot P(A)+\left[\sum_{Z \in \mathbf{Z}_{c}} P(B \mid Z A) \cdot P(Z \mid \bar{A})\right] \cdot P(\bar{A})
\end{aligned}
$$

We can write $P\left(A \rightarrow_{\ell}^{\mathbf{Z}_{c}} B\right)$ by its expansion across the members of $\mathbf{Z}_{c}, Z_{1}, \ldots Z_{n}$, and let $x_{i}$ denote the value of $P\left(B \mid Z_{i} A\right) \cdot P\left(Z_{i}\right)$ for each $Z_{i} \in \mathbf{Z}_{c}$ :

$$
P\left(A \rightarrow \mathbf{Z}_{\ell}^{\mathbf{Z}_{c}} B\right)=\underbrace{P\left(B \mid Z_{1} A\right) \cdot P\left(Z_{1}\right)}_{x_{1}}+\cdots+\underbrace{P\left(B \mid Z_{n} A\right) \cdot P\left(Z_{n}\right)}_{x_{n}}
$$


Since for each $Z \in \mathbf{Z}_{c}$, we calculate $P(Z)$ as follows:

$$
P(Z)=P(Z \mid A) \cdot P(A)+P(Z \mid \bar{A}) \cdot P(\bar{A})
$$

notice that for each $x_{i}$ :

$$
\begin{aligned}
x_{i} & =P\left(B \mid Z_{i} A\right) \cdot\left[P\left(Z_{i} \mid A\right) \cdot P(A)+P\left(Z_{i} \mid \bar{A}\right) \cdot P(\bar{A})\right] \\
& =P\left(B \mid Z_{i} A\right) \cdot P\left(Z_{i} \mid A\right) \cdot P(A)+P\left(B \mid Z_{i} A\right) \cdot P\left(Z_{i} \mid \bar{A}\right) \cdot P(\bar{A})
\end{aligned}
$$

Thus,

$$
x_{1}+\cdots+x_{n}=\left[\sum_{Z \in \mathbf{Z}_{c}} P(B \mid Z A) \cdot P(Z \mid A)\right] \cdot P(A)+\left[\sum_{Z \in \mathbf{Z}_{c}} P(B \mid Z A) \cdot P(Z \mid \bar{A})\right] \cdot P(\bar{A})
$$

hence,

$$
P\left(A \rightarrow \mathbf{Z}_{\ell} B\right)=\left[\sum_{Z \in \mathbf{Z}_{c}} P(B \mid Z A) \cdot P(Z \mid A)\right] \cdot P(A)+\left[\sum_{Z \in \mathbf{Z}_{c}} P(B \mid Z A) \cdot P(Z \mid \bar{A})\right] \cdot P(\bar{A})
$$

But notice that

$$
P(B \mid A)=\sum_{Z \in \mathbf{Z}_{c}} P(B \mid Z A) \cdot P(Z \mid A)
$$

Thus, substituting into (35) yields:

$$
P\left(A \rightarrow \mathbf{Z}_{\ell} B\right)=P(B \mid A) \cdot P(A)+\left[\sum_{Z \in \mathbf{Z}_{c}} P(B \mid Z A) \cdot P(Z \mid \bar{A})\right] \cdot P(\bar{A})
$$

And thus we have established:

$$
P\left(A \rightarrow_{\ell}^{\mathbf{Z}_{c}} B\right)=P\left(A \rightarrow_{c} B\right)
$$

Theorem 3. For any conditional $\ulcorner$ if $\mathrm{A}, \mathrm{B}\urcorner$ and context $c$, if the partition for $\ulcorner$ if $\mathrm{A}, \mathrm{B}\urcorner$ in $c$ is $\left\{E_{c}\right\}$, then $\ulcorner$ if $\mathrm{A}, \mathrm{B}\urcorner$ is Ramsey-friendly in $c$.

Proof. Let $\ulcorner$ if $\mathrm{A}, \mathrm{B}\urcorner$ be an arbitrary conditional and $c$ an arbitrary context. $\ulcorner$ if $\mathrm{A}, \mathrm{B}\urcorner$ is Ramsey-friendly in $c$ iff $P\left(A \rightarrow_{c} B\right)=P(B \mid A)$. Suppose the partition for $\ulcorner$ if $\mathrm{A}, \mathrm{B}\urcorner$ in $c$ is $\left\{E_{c}\right\}$. Then, by Prob: 


$$
\begin{aligned}
& P\left(A \rightarrow_{c} B \mid \bar{A}\right)=\sum_{Z \in \mathbf{Z}_{c}} P(B \mid A Z) \cdot P(Z \mid \bar{A}) \\
& =P(B \mid A)
\end{aligned}
$$

Substituting into (EQ) yields:

$$
\begin{aligned}
& P\left(A \rightarrow_{c} B\right)=P(B \mid A) \cdot P(A)+P(B \mid A) \cdot P(\bar{A}) \\
& =P(B \mid A)
\end{aligned}
$$

Therefore, $\ulcorner$ if $\mathrm{A}, \mathrm{B}\urcorner$ is Ramsey-friendly in $c$.

Theorem 4. $\ulcorner$ if $\mathrm{A}, \mathrm{B}\urcorner$ is Ramsey-violating in $c$ iff for some $Z \in \mathbf{Z}_{c}: P(Z \mid A) \neq P(Z \mid \bar{A})$ (where $\mathbf{Z}_{c}$ is the partition for $\ulcorner$ if $\mathrm{A}, \mathrm{B}\urcorner$ in $c$ ).

Left-to-right. By Centering plus (EQ):

$$
P\left(A \rightarrow_{c} B\right)=P(B \mid A) \cdot P(A)+P\left(A \rightarrow_{c} B \mid \bar{A}\right) \cdot P(\bar{A})
$$

Therefore,

$$
P\left(A \rightarrow_{c} B\right) \neq P(B \mid A) \text { only if } P(B \mid A) \neq P\left(A \rightarrow_{c} B \mid \bar{A}\right)
$$

So by Prob,

$$
P\left(A \rightarrow_{c} B\right) \neq P(B \mid A) \text { only if } P(B \mid A) \neq P\left(A \rightarrow_{c} B \mid \bar{A}\right)=\sum_{Z \in \mathbf{Z}_{c}} P(B \mid Z A) \cdot P(Z \mid \bar{A})
$$

Finally, notice that (by the law of total probability),

$$
P(B \mid A)=\sum_{Z \in \mathbf{Z}_{c}} P(B \mid Z A) \cdot P(Z \mid A)
$$

Therefore,

$$
P\left(A \rightarrow_{c} B\right) \neq P(B \mid A) \text { only if } \exists Z \in \mathbf{Z}_{c}: P(Z \mid A) \neq P(Z \mid \bar{A})
$$

Right-to-left. Suppose that the background partition for $\ulcorner$ if $\mathrm{A}, \mathrm{B}\urcorner, \mathbf{Z}_{c}$, is such that there is some $Z \in \mathbf{Z}_{c}: P(Z \mid A) \neq P(Z \mid \bar{A})$. From Centering and (EQ) again:

$$
P\left(A \rightarrow_{c} B\right)=P(B \mid A) \cdot P(A)+P\left(A \rightarrow_{c} B \mid \bar{A}\right) \cdot P(\bar{A})
$$


Therefore,

$$
\text { If } P(B \mid A) \neq P\left(A \rightarrow_{c} B \mid \bar{A}\right) \text { then } P\left(A \rightarrow_{c} B\right) \neq P(B \mid A)
$$

So by Prob:

$$
\text { If } P(B \mid A) \neq \sum_{Z \in \mathbf{Z}_{c}} P(B \mid Z A) \cdot P(Z \mid \bar{A}) \text {, then } P\left(A \rightarrow_{c} B\right) \neq P(B \mid A)
$$

So by the law of total probability:

$$
\text { If } \sum_{Z \in \mathbf{Z}_{c}} P(B \mid Z A) \cdot P(Z \mid A) \neq \sum_{Z \in \mathbf{Z}_{c}} P(B \mid Z A) \cdot P(Z \mid \bar{A}) \text {, then } P\left(A \rightarrow_{c} B\right) \neq P(B \mid A)
$$

But furthermore,

$$
\text { If } \exists Z \in \mathbf{Z}_{c}: P(Z \mid A) \neq P(Z \mid \bar{A}) \text {, then } \sum_{Z \in \mathbf{Z}_{c}} P(B \mid Z A) \cdot P(Z \mid A) \neq \sum_{Z \in \mathbf{Z}_{c}} P(B \mid Z A) \cdot P(Z \mid \bar{A})
$$

So since $\exists Z \in \mathbf{Z}_{c}: P(Z \mid A) \neq P(Z \mid \bar{A})$ (by assumption), it follows that

$$
\sum_{Z \in \mathbf{Z}_{c}} P(B \mid Z A) \cdot P(Z \mid A) \neq \sum_{Z \in \mathbf{Z}_{c}} P(B \mid Z A) \cdot P(Z \mid \bar{A})
$$

and hence that $P\left(A \rightarrow_{c} B\right) \neq P(B \mid A)$, and hence that $\ulcorner$ if $\mathrm{A}, \mathrm{B}\urcorner$ is Ramsey-violating in $c$.

\section{Proofs of facts}

In each of these statements of facts and proofs, I assume that $A, B$ are the propositions expressed by the sentences A and B in the relevant context.

Fact 1 (Modus ponens). For all $c: A \rightarrow_{c} B, A \models B$

Proof. Suppose $w \in A \rightarrow_{c} B$ and $w \in A$, for an arbitrary world $w$ and context $c$. By Stalnaker semantics, $w^{\prime} \in B$, where $w^{\prime}$ is the $c$-closest $A$-world to $w$. Since $w \in A$, by Centering, the $c$-closest $A$-world to $w$ is $w$. Therefore, $w \in B .{ }^{48}$

\footnotetext{
${ }^{48}$ For a version of this sort of semantics that predicts failures of modus ponens for embedded conditionals (a possibility raised by [mcgee:1985]), see [kratzer:1986, kratzer:2012, khoo:2013a].
} 
Fact 2 (Strong centering). For all $c: A \rightarrow_{c} B$ is strongly centered in $c$

Proof. Suppose that $w \in A$ for an arbitrary world $w$ and context $c$. Then by Stalnaker semantics, $w \in A \rightarrow_{c} B$ iff $w^{\prime} \in B$, where $w^{\prime}$ is the $c$-closest $A$-world to $w$. Since $w \in A$, by Centering, the $c$-closest $A$-world to $w$ is $w$. Therefore, $w \in A \rightarrow_{c} B$ iff $w \in B$. Therefore, $A$ entails that $B$ and $A \rightarrow_{c} B$ are equivalent, which entails that $A \rightarrow_{c} B$ is strongly centered in $c$, and this holds for all $c$ since the choice of $c$ was arbitrary.

Fact 3 (Strength). For all $c: A \rightarrow_{c} B \models(A \supset B) ;(A \supset B) \not \models A \rightarrow_{c} B$

Proof that $A \rightarrow_{c} B \models(A \supset B)$. Suppose for reductio that there is a $w \in A \rightarrow_{c} B$ such that $w \notin A \supset B$. Then $w \in A$ and $w \notin B$. Since $w \in A \rightarrow{ }_{c} B$, by Stalnaker semantics, $w^{\prime} \in B$, where $w^{\prime}$ is the $c$-closest $A$-world to $w$. Since $w \in A$, by Centering, the $c$-closest $A$-world to $w$ is $w$. Therefore, $w \in B$. Contradiction. Therefore, $A \rightarrow_{c} B \mid=(A \supset B)$.

Proof that $(A \supset B) \forall \neq A \rightarrow_{c} B$. To show this it is sufficient to show that there is a world $w \in(A \supset B)$ that is not in $A \rightarrow_{c} B$, for some $c$. Suppose $w \notin A$. Then $w \in(A \supset B)$. But we may suppose that $w^{\prime} \notin B$, where $w^{\prime}$ is the $c$-closest $A$-world to $w$; all this requires given Serious partitioning is that the unique $A$-world in $[w] \mathbf{z}_{c}$ is not in $B$. Therefore, there is a world $w \in(A \supset B)$ that is not in $A \rightarrow_{c} B$, for some $c$.

\section{Partition standoffs}

Let $\ulcorner$ if $\mathrm{A}, \mathrm{B}\urcorner$ be some conditional and $c$ and $c^{\prime}$ be contexts differing at most from each other in the partition they assign to this conditional. Let $\mathbf{Z}$ be the partition assigned it by $c$ and $\mathbf{Y}$ be the partition assigned it by $c^{\prime}$; remember, both $\mathbf{Z}$ and $\mathbf{Y}$ will partition the same space, $E_{c}=E_{c^{\prime}}$. But since $\mathbf{Z} \neq \mathbf{Y}, A \rightarrow_{c} B \neq A \rightarrow_{c^{\prime}} B$. I show here that my theory will predict that it is possible that for some $Z \in \mathbf{Z}$ and $Y \in \mathbf{Y}$, where $Z Y \neq \emptyset$ : $P\left(A \rightarrow_{c} B \mid Z\right)=1$ and $P\left(A \rightarrow_{c} B \mid Y\right)=0$. Thus, my theory will predict, indirectly, for every world $w \in Z Y$, that $A \rightarrow_{c} B$ is true at $w$ and $A \rightarrow_{c^{\prime}} B$ is false at $w$.

Here's how to construct such a case schematically.

- Let $A Z \models B$ and $A Y \models \bar{B}$.

- This is sufficient to ensure that $P(B \mid A Z)=1$ and $P(B \mid A Y)=0$. 
- This is sufficient to ensure that $P\left(A \rightarrow_{c} B \mid Z\right)=1$ and $P\left(A \rightarrow_{c^{\prime}} B \mid Y\right)=0$.

- But since $Z Y \neq \emptyset$, there must be some $w \in Z Y$ such that $A \rightarrow_{c} B$ is true at $w$ and $A \rightarrow c^{\prime} B$ is false at $w$.

Here's a non-schematic scenario with these properties.

Cancer. Zed is in Australia watching TV and Yanni is in the UK watching TV. They happen to be watching the same simultaneously-broadcast documentary about smoking and lung cancer. One of the people featured in the documentary is named John, whom the viewers slowly learn more about as the show progresses. So far, the show has revealed that all the people in a small sample of patients (of which John is a member) who smoke and have gene $\mathrm{Z}$ have cancer, and that none of the smokers in that group who have gene $Y$ have cancer. It has also revealed that John very likely has both genes (and hence very likely that John doesn't smoke), but not yet whether John smokes. Zed saw the part about gene $\mathrm{Z}$ but happened to miss the part about gene $\mathrm{Y}$ (he was answering a phone call); while Yanni saw the part about gene $\mathrm{Y}$ but missed the part about gene $\mathrm{Z}$ (he was in the bathroom). In the commercial break just prior to the big reveal about whether John smokes and has cancer, both Zed and Yanni are considering the likelihood of the following conditional:

If John smokes, he has cancer.

Zed reasons as follows: Either John has Z or he doesn't. Suppose he has Z. Then (52) is certainly true (since everyone with $\mathrm{Z}$ who smokes has cancer). Suppose he doesn't have Z. Then (52) is uncertain. But since it's likely John has Z, (52) is likely.

Yanni reasons as follows: Either John has Y or he doesn't. Suppose he has $\mathrm{Y}$. Then (52) is certainly false (since no one with $\mathrm{Y}$ who smokes has cancer). Suppose he doesn't have Y. Then (52) is uncertain. But since it's likely John has $\mathrm{Y},(52)$ is unlikely.

I submit that Zed and Yanni's reasoning here is impeccable, yet they come to contradictory conclusions - it is analogous to a "Gibbardian standoff" case (cf. [gibbard:1981]). ${ }^{49} \mathrm{My}$ theory predicts that (52) has a different semantic value in their two contexts, due to the

\footnotetext{
${ }^{49}$ One issue with standoff cases is whether the two individuals would thereby disagree if they talked to one another. This is a matter I set aside since it goes beyond the scope of the paper. See [gibbard:1981, edgington:1995, bennett:2003, williams:2008, stalnaker:2011] for further discussion.
} 
fact that different questions are under discussion in them ( $\mathbf{Z}$ in Zed's context and $\mathbf{Y}$ in Yanni's context). I think this is a plausible prediction.

\section{References}

[adams:1970] Ernest Adams. Subjunctive and indicative conditionals. Foundations of Language, 6(1):89-94, 1970.

[adams:1975] Ernest Adams. The Logic of Conditionals, volume 86 of Synthese Library. D. Reidel, Boston, 1975.

[barhillel:1980] Maya Bar-Hillel. The base-rate fallacy in probability judgments. Acta Psychologica, 44:211-233, 1980.

[barker:1998] Stephen J. Barker. Predetermination and tense probabilism. Analysis, 58(4):290-296, 1998.

[bennett:2003] Jonathan Bennett. A Philosophical Guide to Conditionals. Oxford University Press, Oxford, 2003.

[bradley:2012] Richard Bradley. Multidemonsional possible-world semantics for conditionals. Philosophical Review, 121(4):539-571, 2012.

[chalmers:2007] David Chalmers and Alan Hájek. Ramsey + moore $=$ god. Analysis, 67(72):170-172, 2007.

[douven:2008] Igor Douven. Kaufmann on the probabilities of conditionals. Journal of Philosophical Logic, 37(3):259-266, 2008.

[douven:2013] Igor Douven. The probabilities of conditionals revisited. Cognitive Science, pages 1-20, 2013.

[dowell:2011] Janice Dowell. A flexible contextualist account of epistemic modals. Philosophers' Imprint, 11(14):1-25, 2011.

[edgington:1991] Dorothy Edgington. The mystery of the missing matter of fact. Proceedings of the Aristotelian Society, Supplementary Volumes, 65:185-209, 1991.

[edgington:1995] Dorothy Edgington. On conditionals. Mind, 104:235-329, 1995.

[edgington:1997] Dorothy Edgington. Truth, objectivity, counterfactuals, and gibbard. Mind, 106(421):107-116, 1997. 
[edgington:2004] Dorothy Edgington. Counterfactuals and the benefit of hindsight. In Cause and Chance: Causation in an Indeterministic World, pages 12-28. Routledge, New York, 2004.

[ellis:1978] Brian Ellis. A unified theory of conditionals. Journal of Philosophical Logic, 7(1):107-124, 1978.

[evans:2004] Jonathan St. B. T. Evans and David E. Over. If. Oxford University Press, Oxford, 2004.

[fraassen:1976] Bas van Fraassen. Probabilities of conditionals. In William L. Harper and C. Hooker, editors, Foundations of Probability Theory, Statistical Inference, and Statistical Theories of Science, pages 261-300. Reidel, Dordrecht, 1976.

[fraassen:1980] Bas van Fraassen. Review of brian ellis, rational belief systems. Canadian Journal of Philosophy, 10:497-511, 1980.

[frank:1997] Anette Frank. Context Dependence in Modal Constructions. PhD thesis, University of Stuttgart, 1997.

[gardenfors:1988] Peter Gardenfors. Knowledge in Flux: Modeling the Dynamics of Epistemic States. MIT Press, Cambridge, 1988.

[geurts:2004] Bart Geurts. On an ambiguity in quantified conditionals. http://ncs . ruhosting.nl/bart/papers/conditionals.pdf, 2004.

[geurts:2005] Bart Geurts. Entertaining alternatives: Disjunctions as modals. Natural Language Semantics, 13:383-410, 2005.

[gibbard:1981] Allan Gibbard. Two recent theories of conditionals. In William L. Harper, Robert Stalnaker, and Glenn Pearce, editors, Ifs, pages 211-247. Reidel, Dordrecht, 1981.

[gigerenzer:1995] Gerd Gigerenzer and Ulrich Hoffrage. How to improve bayesian reasoning without instruction: Frequency formats. Psychological Review, 102(4):684-704, 1995 .

[glanzberg:2007] Michael Glanzberg. Context, content, and relativism. Philosophical Studies, 136:1-29, 2007.

[groenendijk:1984] Jeroen Groenendijk and Martin Stokhof. Studies on the Semantics of Questions and Studies on the Semantics of Questions and Studies in the Semantics of Questions and the Pragmatics of Answers. PhD thesis, University of Amsterdam, 1984. 
[groenendijk:1997] Jeroen Groenendijk and Martin Stokhof. Questions. In Johan van Benthem and Alice ter Meulen, editors, Handbook of Logic and Language, pages 1055-1124. Elsevier, 1997.

[hajek:1989] Alan Hájek. Probabilities of conditionals - revisited. Journal of Philosophical Logic, 18:423-428, 1989.

[hajek:1994] Alan Hájek. Triviality on the cheap? In E. Eells and B. Skyrms, editors, Probability and Conditionals: Belief Revision and Rational Decision, pages 113140. Cambridge University Press, Cambridge, 1994.

[hajek:2011b] Alan Hájek. Triviality pursuit. Topoi, 30:3-15, 2011.

[hajek:2012] Alan Hájek. The fall of "adams' thesis"? Journal of Logic, Language, and Information, 21:145-161, 2012.

[hajekhall:1994] Alan Hájek and Ned Hall. The hypothesis of the conditional construal of conditional probability. In Ellery Eells and Brian Skyrms, editors, Probability and Conditionals, chapter 6, pages 75-111. Cambridge University Press, Cambridge, 1994.

[hawthorne:2005] John Hawthorne. Chance and counterfactuals. Philosophy and Phenomenological Research, 70(2):396-405, 2005.

[hoffrage:2000] Ulrich Hoffrage, Samuel Lindsey, Ralph Hertwig, and Gerd Gigerenzer. Communicating statistical information. Science, 290(5500):2261-2262, 2000.

[jackson:1979] Frank Jackson. On assertion and indicative conditionals. Philosophical Review, 88:565-89, 1979.

[jackson:1987] Frank Jackson. Conditionals. Blackwell, Oxford, 1987.

[jeffrey:1991] Richard Jeffrey. Matter-of-fact conditionals. Aristotelian Society: Supplemental Volume, 65:161-183, 1991.

[kahneman:1973] Daniel Kahneman and Amos Tversky. On the psychology of prediction. Psychological Review, 80:237-251, 1973.

[kahneman:1982] Daniel Kahneman and Amos Tversky. Evidence impact of base rates. In Daniel Kahneman, Paul Slovic, and Amos Tversky, editors, Judgment Under Uncertainty: Heuristics and Biases, pages 153-160. Cambridge University Press, Cambridge, 1982.

[kaufmann:2004] Stefan Kaufmann. Conditioning against the grain: Abduction and indicative conditionals. Journal of Philosophical Logic, 33:583-606, 2004. 
[kaufmann:2005b] Stefan Kaufmann. Conditional predictions. Linguistics and Philosophy, 28:181-231, 2005.

[kaufmann:2009a] Stefan Kaufmann. Conditionals right and left: Probabilities for the whole family. Journal of Philosophical Logic, 38:1-53, 2009.

[khoo:2013a] Justin Khoo. A note on gibbard's proof. Philosophical Studies, 166(1):153$164,2013$.

[khoo:2013c] Justin Khoo. Conditionals, indeterminacy, and triviality. Philosophical Perspectives, 27(1):260-287, 2013.

[king:2013a] Jeffrey C. King. Supplementives, the coordination account, and conflicting intentions. Philosophical Perspectives, 27:288-311, 2013.

[king:2013b] Jeffrey C. King. The metasemantics of contextual sensitivity. In A. Burgess and B. Sherman, editors, New Essays in Metasemantics. Oxford University Press, 2013.

[kratzer:1977] Angelika Kratzer. What 'must' and 'can' must and can mean. Linguistics and Philosophy, 1:337-355, 1977.

[kratzer:1981] Angelika Kratzer. The notional category of modality. In H. J. Eikmeyer and H. Rieser, editors, Words, Worlds, and Contexts. New Approaches in Words Semantics, pages 38-74. de Gruyter, Berlin, 1981.

[kratzer:1986] Angelika Kratzer. Conditionals. Chicago Linguistics Society, 22(2):1-15, 1986.

[kratzer:1991] Angelika Kratzer. Modality. In Arnim von Stechow and Dieter Wunderlich, editors, Handbuch Semantik, chapter 23, pages 639-650. de Gruyter, Berlin and New York, 1991.

[kratzer:2012] Angelika Kratzer. Collected Papers on Modals and Conditionals. Oxford University Press, Oxford, 2012.

[lewis:1976] David Lewis. Probabilities of conditionals and conditional probabilities. The Philosophical Review, 85:297-315, 1976.

[lewis:1981c] David Lewis. Causal decision theory. Australasian Journal of Philosophy, 59:5-30, 1981.

[lewis:1986] David Lewis. On the Plurality of Worlds. Blackwell, Oxford, 1986.

[lewis:1986a] David Lewis. Probabilities of conditionals and conditional probabilities ii. The Philosophical Review, 95:581-589, October 1986. 
[mcdermott:1999] Michael McDermott. Counterfactuals and access points. Mind, 108:291334, 1999.

[mcgee:1985] Vann McGee. A counterexample to modus ponens. The Journal of Philosophy, 82(9):462-471, 1985.

[mcgee:1989] Vann McGee. Conditional probabilities and compounds of conditionals. Philosophical Review, 98:485-542, 1989.

[mcgee:2000] Vann McGee. To tell the truth about conditionals. Analysis, 60(1):107-111, 2000 .

[moss:2012] Sarah Moss. On the pragmatics of counterfactuals. Nous, 46(2):561-86, 2012.

[moss:2015] Sarah Moss. On the semantics and pragmatics of epistemic vocabulary. Semantics \& Pragmatics, 8(5):1-81, 2015.

[perry:2009] John Perry. Directing intentions. In J. Almog and P. Leonardi, editors, The Philosophy of David Kaplan, pages 187-201. Oxford University Press, Oxford, 2009 .

[pollock:1981] John L. Pollock. A refined theory of counterfactuals. Journal of Philosophical Logic, 10:239-266, 1981.

[ramsey:1931] Frank P. Ramsey. The Foundations of Mathematics and other Logical Essays. Kegan Paul, Trench, Trubner \& Co., London, 1931.

[roberts:1996b] Craige Roberts. Information structure in discourse: Towards an integrated formal theory of pragmatics. In Jae Hak Yoon and Andreas Kathol, editors, Ohio State University Working Papers in Linguistics, volume 49. 1996.

[roberts:2012a] Craige Roberts. Information structure in discourse: Towards an integrated formal theory of pragmatics. Semantics \& Pragmatics, 5(6):1-69, 2012.

[roberts:2012b] Craige Roberts. Information structure: Afterword. Semantics 85 Pragmat$i c s, 5(7): 1-19,2012$.

[rothschild:2013a] Daniel Rothschild. Do indicative conditionals express propositions? Nous, 47(1):49-68, 2013.

[schulz:2014a] Moritz Schulz. Counterfactuals and arbitrariness. Mind, 123(492):10211055, 2014.

[skyrms:1988] Brian Skyrms. Conditional chance. In J. H. Fetzer, editor, Probability and Causality, Studies in Epistemology, Logic, Methodology, and the Philosophy of Science, pages 161-178. D. Reidel, 1988. 
[skyrms:1990] Brian Skyrms. The Dynamics of Rational Deliberation. Harvard University Press, Cambridge, 1990.

[sorensen:1988a] Roy Sorensen. Blindspots. Oxford University Press, Oxford, 1988.

[sorensen:2001] Roy Sorensen. Vagueness and Contradiction. Oxford University Press, Oxford, 2001.

[stalnaker:1968] Robert Stalnaker. A theory of conditionals. In N. Rescher, editor, Studies in Logical Theory, pages 98-112. Oxford University Press, Oxford, 1968.

[stalnaker:1970] Robert Stalnaker. Probability and conditionals. Philosophy of Science, 37(1):64-80, 1970.

[stalnaker:1975] Robert Stalnaker. Indicative conditionals. Philosophia, 5:269-86, 1975.

[stalnaker:1976] Robert Stalnaker. Possible worlds. Nous, 10:65-75, 1976.

[stalnaker:1978] Robert Stalnaker. Assertion. In P. Cole, editor, Syntax and Semantics 9: Pragmatics, pages 315-332. Academic Press, New York, 1978.

[stalnaker:1980] Robert Stalnaker. A defense of conditional excluded middle. In William L. Harper, Glenn Pearce, and Robert Stalnaker, editors, Ifs. Reidel, Dordrecht, 1980 .

[stalnaker:1984] Robert Stalnaker. Inquiry. MIT Press, 1984.

[stalnaker:1994] Robert Stalnaker and Richard Jeffrey. Conditionals as random variables. In Ellery Eells and Brian Skyrms, editors, Probabilities and Conditionals: Belief Revision and Rational Decision, pages 31-46. Cambridge University Press, Cambridge, 1994.

[stalnaker:2011] Robert Stalnaker. Conditional propositions and conditional assertions. In Andy Egan and Brian Weatherson, editors, Epistemic Modality. Oxford University Press, Oxford, 2011.

[starr:2014b] William B. Starr. What 'if'? Philosophers' Imprint, 14(10):1-27, 2014.

[warmbrod:1983] Ken Warmbrod. Epistemic conditionals. Pacific Philosophical Quarterly, 64:249-265, 1983.

[willer:2010] Malte Willer. New surprises for the ramsey test. Synthese, 2010.

[williams:2008] J. Robert G. Williams. Conversation and conditionals. Philosophical Studies, 138:211-223, 2008.

[williamson:1994] Timothy Williamson. Vagueness. Routledge, New York, 1994. 